\title{
Small Seifert fibered surgery on hyperbolic pretzel knots
}

\author{
JEFFREY MEIER
}

\begin{abstract}
We complete the classification of hyperbolic pretzel knots admitting Seifert fibered surgeries. This is the final step in understanding all exceptional surgeries on hyperbolic pretzel knots. We also present results toward similar classifications for nonpretzel Montesinos knots of length three.
\end{abstract}

57M25; 57M50

\section{Introduction}

The study of exceptional surgery on hyperbolic knots has been well developed over the last quarter century. One particularly well studied problem is that of exceptional surgery on arborescent knots, which include Montesinos knots and pretzel knots. Thanks to the positive solution to the geometrization conjecture by Perelman $[38 ; 39 ; 40]$, any exceptional surgery is either reducible, toroidal, or a small Seifert fibered space. Exceptional surgeries on hyperbolic arborescent knots of length 4 or greater have been classified by $\mathrm{Wu}$ [52], as have exceptional surgeries on hyperbolic 2-bridge knots; see Brittenham and $\mathrm{Wu}$ [7]. It has been shown that no hyperbolic arborescent knot admits a reducible surgery (see $\mathrm{Wu}$ [48]), and toroidal surgeries on hyperbolic arborescent knots of length three are completely classified; see $\mathrm{Wu}$ [51].

Therefore, it only remains to understand small Seifert fibered surgeries on Montesinos knots of length three. Furthermore, finite surgeries on Montesinos knots only occur in two instances, along two slopes of each of the pretzel knots $P(-2,3,7)$ and $P(-2,3,9)$; see Futer, Ishikawa, Kabaya, Mattman and Shimokawa [16] and Ichihara and Jong [23]. Thus, one must only consider nonfinite, atoroidal Seifert fibered surgeries on hyperbolic Montesinos knots of length three.

According to $\mathrm{Wu}[46 ; 45]$, the only hyperbolic Montesinos knots of length three that are pretzel knots and might admit Seifert fibered surgeries have the form $P\left(q_{1}, q_{2}, q_{3}\right)$ or $P\left(q_{1}, q_{2}, q_{3},-1\right)$, where $\left(\left|q_{1}\right|,\left|q_{2}\right|,\left|q_{3}\right|\right)=\left(2,\left|q_{2}\right|,\left|q_{3}\right|\right),\left(3,3,\left|q_{3}\right|\right)$ or $(3,4,5)$, and in the length four case, then $q_{i}>0$ for $i=1,2,3$. Recently, it was shown that hyperbolic pretzel knots of the form $P(p, q, q)$ with $p, q$ positive (see Ichihara and 
Jong [22]) or $P(-2, p, p)$ with $p$ positive (see Ichihara, Jong and Kabaya [24]) do not admit Seifert fibered surgeries.

Further work by $\mathrm{Wu}[46 ; 52 ; 45]$ tells us that if a nonpretzel Montesinos knot admits a small Seifert fibered surgery, then it has one of the following forms: $K[1 / 3,-2 / 3,2 / 5]$, $K[-1 / 2,1 / 3,2 /(2 a+1)]$ for $a \in\{3,4,5,6\}$, or $K[-1 / 2,1 /(2 q+1), 2 / 5]$ for $q \geq 1$.

In this paper, we address the issue of which of the above listed Montesinos knots admit small Seifert fibered surgeries. The main results are stated below. Keep in mind that there is an orientation reversing homeomorphism $K(\alpha)=\bar{K}(-\alpha)$, where $\bar{K}$ is the mirror of $K$. Thus, we often consider in our analysis, and present in our results, only one representative of $\{K, \bar{K}\}$.

For the following theorem, recall that the pretzel knot $P(p, q, r)$ with $|p|,|q|,|r| \geq 2$ is hyperbolic unless it is either $P(-2,3,3)$ or $P(-2,3,5)$, in which case it is the torus knot $T(3,4)$ or $T(3,5)$, respectively; see Oertel [37]. Below, when we consider the knots $P(-2,2 p+1,2 q+1)$, we will assume that $|p|<|q|$ when $p$ and $q$ have the same sign and that $p>0$ when their signs differ.

Theorem 1.1 The hyperbolic pretzel knot $P(-2,2 p+1,2 q+1)$, with the conventions discussed above, admits a small Seifert fibered surgery if and only if $p=1$, in which case it admits precisely the following small Seifert fibered surgeries:

- $P(-2,3,2 q+1)(4 q+6)=S^{2}(1 / 2,-1 / 4,2 /(2 q-5))$

- $P(-2,3,2 q+1)(4 q+7)=S^{2}(2 / 3,-2 / 5,1 /(q-2))$

Theorem 1.2 Hyperbolic pretzel knots of the form $P(3,3, m)$ or $P(3,3,2 m,-1)$ admit no small Seifert fibered surgeries. Pretzel knots of the form $P(3,-3, m)$, with $m>1$, admit small Seifert fibered surgeries precisely in the following cases:

- $P(3,-3,2)(1)=S^{2}(1 / 3,1 / 4,-3 / 5)$

- $P(3,-3,3)(1)=S^{2}(1 / 2,-1 / 5,-2 / 7)$

- $P(3,-3,4)(1)=S^{2}(-1 / 2,1 / 5,2 / 7)$

- $P(3,-3,5)(1)=S^{2}(2 / 3,-1 / 4,-2 / 5)$

- $P(3,-3,6)(1)=S^{2}(1 / 2,-2 / 3,2 / 13)$

Theorem 1.3 The pretzel knots $P(3, \pm 4, \pm 5)$ and $P(3,4,5,-1)$ admit no small Seifert fibered surgeries. 
Theorem 1.4 Suppose that $K$ is a nonpretzel Montesinos knot and $K(\alpha)$ is a small Seifert fibered space. Then either $K=K[-1 / 2,2 / 5,1 /(2 q+1)]$ for some $q \geq 5$, or $K$ is on the following list and has the described surgeries:

- $K[1 / 3,-2 / 3,2 / 5](-5)=S^{2}(1 / 4,2 / 5,-3 / 5)$

- $K[-1 / 2,1 / 3,2 / 7](-1)=S^{2}(1 / 3,1 / 4,-4 / 7)$

- $K[-1 / 2,1 / 3,2 / 7](0)=S^{2}(1 / 2,3 / 10,-4 / 5)$

- $K[-1 / 2,1 / 3,2 / 7](1)=S^{2}(1 / 2,1 / 3,-16 / 19)$

- $K[-1 / 2,1 / 3,2 / 9](2)=S^{2}(1 / 2,-1 / 3,-3 / 20)$

- $K[-1 / 2,1 / 3,2 / 9](3)=S^{2}(1 / 2,-1 / 5,-3 / 11)$

- $K[-1 / 2,1 / 3,2 / 9](4)=S^{2}(-1 / 4,2 / 3,-3 / 8)$

- $K[-1 / 2,1 / 3,2 / 11](-2)=S^{2}(-2 / 3,2 / 5,2 / 7)$

- $K[-1 / 2,1 / 3,2 / 11](-1)=S^{2}(-1 / 2,-2 / 7,2 / 9)$

- $K[-1 / 2,1 / 3,2 / 5](3)=S^{2}(1 / 2,-1 / 3,-2 / 15)$

- $K[-1 / 2,1 / 3,2 / 5](4)=S^{2}(1 / 2,-1 / 6,-2 / 7)$

- $K[-1 / 2,1 / 3,2 / 5](5)=S^{2}(-1 / 3,-1 / 5,3 / 5)$

- $K[-1 / 2,1 / 5,2 / 5](7)=S^{2}(1 / 2,-1 / 5,-2 / 9)$

- $K[-1 / 2,1 / 5,2 / 5](8)=S^{2}(-1 / 4,3 / 4,-2 / 5)$

- $K[-1 / 2,1 / 7,2 / 5](11)=S^{2}(-1 / 3,3 / 4,-2 / 7)$

Each of the theorems stated above is proved below using a common procedure. First, we exploit the symmetries of the Montesinos knots in question to describe the surgery space as a branched double cover of a link. Next, we use rational tangle filling theory and exceptional surgery bounds to restrict our attention to a finite list of such links, ie, we restrict the parameters for which the Montesinos knots in question can admit small Seifert fibered surgeries. Finally, we use knot theory invariants to show that the branched double covers of links on this finite list cannot be Seifert fibered (excepting, of course, the cases that are). This last step makes use of the Mathematica ${ }^{\circledR}$ package KnotTheory [43].

It should be noted that, concurrent with the preparation of this paper, the author learned that similar results had been obtained by $\mathrm{Wu}$, though using different techniques. $\mathrm{Wu}$ also restricts the families to finite families of surgery spaces, but does so by studying exceptional surgery on tubed Montesinos knots (see [44]). He then appeals to the computer program Snappex, to determine the hyperbolic structure of the surgeries in question (see [47]). 


\subsection{A word on nonintegral surgeries}

In a survey by $\mathrm{Wu}$ [49], it is shown how techniques and results from Brittenham [6] and $\mathrm{Wu}$ [50] can be combined with work of Delman [13] to study which length three Montesinos knots have exteriors that admit persistent essential laminations.

Theorem 1.5 Let $K$ be a hyperbolic Montesinos knot of length three. Then the exterior of $K$ admits a persistent essential lamination, and, thus, cannot admit a nonintegral small Seifert fibered surgery, unless $K=K[x, 1 / p, 1 / q]$ (or its mirror image), where $x \in\{-1 /(2 n),-1 \pm 1 /(2 n),-2+1 /(2 n)\}$, and $p, q$ and $n$ are positive integers.

With this in mind, for many of the families of pretzel knots considered in this paper, it is only necessary to consider integral surgeries. However, for some families, it is necessary to consider nonintegral surgeries. To be specific, of all the pretzel knots considered in this paper, only the following families could potentially admit nonintegral small Seifert fibered surgeries:

- $P(-2,2 p+1,2 q+1)$ with $1 \leq p<q$

- $\quad P(3,3,-2 m)$ with $m \geq 2$

- $K[-1 ; 1 / 3,1 / 3,1 / 2 m]$ with $m \geq 1$

- $P(3,-4,5)$ or $P(3,4,5,-1)$

Thus, whenever such a family is considered, we have shown that, in fact, there are no nonintegral small Seifert fibered surgeries. One of the biggest open problems in the study of exceptional Dehn surgery is the following conjecture (see Gordon [19]).

Conjecture 1.6 Any Seifert fibered surgery on a hyperbolic knot is integral.

The results of this paper are the final steps of an affirmative answer to Conjecture 1.6 in the case of hyperbolic arborescent knots.

Theorem 1.7 Any Seifert fibered surgery on a hyperbolic arborescent knot is integral.

\subsection{Open questions}

Unfortunately, the techniques of this paper are insufficient to complete the classification of Seifert fibered surgery on Montesinos knots. We are left with the following question, which is the final step in a complete classification of exceptional surgery on arborescent knots. 
Question 1.8 Do the Montesinos knots $K[-1 / 2,2 / 5,1 /(2 q+1)]$ with $q \geq 5$ admit small Seifert fibered surgeries? ${ }^{1}$

Organization Section 2 presents general background material and outlines how knot invariants will be used to obstruct small Seifert fibered surgeries. Sections 3, 4, 5 and 6 present, respectively, the proofs of Theorems 1.1, 1.2, 1.3 and 1.4.

Acknowledgements The author would like to thank his advisor, Cameron Gordon, for many conversations full of insightful advice, patience, and encouragement. The author is also grateful to Ying-Qing Wu for his friendly correspondence regarding the nature of this problem, and to the referee for his or her careful reading of the manuscript and helpful comments. This work was supported by NSF grant number DMS-0636643.

\section{Preliminaries}

\subsection{Dehn surgery}

Let $K$ be a knot in $S^{3}$ and let $N(K)$ be a regular neighborhood of $K$. We let $M_{K}=\overline{S^{3} \backslash N(K)}$ be the exterior of $K$. The set of isotopy classes of simple closed curves on $\partial N(K)=\partial M_{K}$ is in bijection with $H_{1}\left(\partial M_{K}\right)$, the latter of which is naturally generated by two elements $[\mu]$ and $[\lambda]$, where $[\mu]$ generates $H_{1}\left(M_{K}\right) \cong \mathbb{Z}$, $[\lambda]=0 \in H_{1}\left(M_{K}\right)$, and $\mu$ and $\lambda$ intersect geometrically once on $\partial M_{K}$. Orient $\mu$ and $\lambda$ so that $\mu \cdot \lambda=+1$. The unoriented isotopy class of a simple closed curve $\gamma \subset \partial M_{K}$ is called a slope and can be thought of as an element $m / l \in \mathbb{Q} \cup\{\infty\}$, where $[\gamma]=m[\mu]+l[\lambda]$ in $H_{1}\left(\partial M_{K}\right)$. The curves $\mu$ and $\lambda$ are called the meridian and the longitude, respectively.

Given two slopes $\alpha$ and $\beta$ on $T^{2}$, let the distance between $\alpha$ and $\beta, \Delta(\alpha, \beta)$ be their minimal geometric intersection number. If $\alpha=m / l$ and $\beta=m^{\prime} / l^{\prime}$, then we have $\Delta(\alpha, \beta)=\left|m l^{\prime}-m^{\prime} l\right|$.

Let $V$ be a solid torus, and let $\varphi: \partial V \rightarrow \partial M_{K}$ be a homeomorphism which takes the meridian of $V$ to a slope $\alpha$ on $\partial M_{K}$. Then Dehn surgery on $K$ along $\alpha$, or $\alpha$-Dehn surgery on $K$, is the space $K(\alpha)=M_{K} \cup \varphi V$; see Figure 1. For a general overview of the theory of Dehn surgery, a subject that has been well-studied since its introduction by Dehn in 1910 [12]; see [19].

${ }^{1}$ Question 1.8 has recently been answered in the negative by Ichihara and Masai [25]. Their work uses a computer algorithm to verify the hyperbolic structures of surgeries on these knots. Together, their work and the present results complete the classification of exceptional surgeries on arborescent knots. 

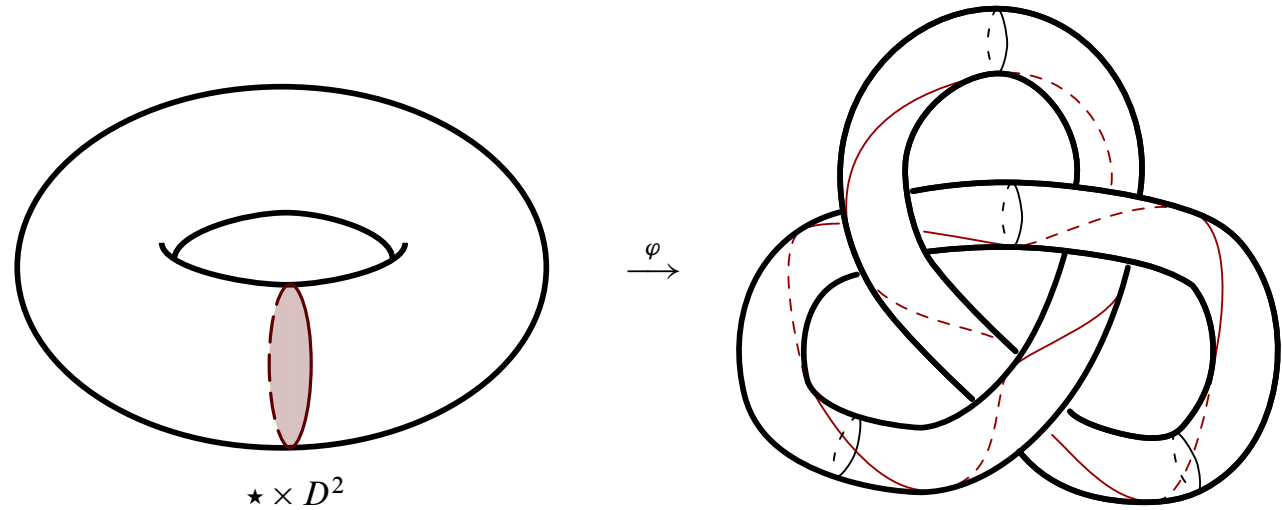

Figure 1: On the right, we see the exterior, $M_{K}$, of the left handed trefoil. The surgery space $K(0)$ is formed by filling the boundary of $M_{K}$ with a solid torus such that the meridian maps to a 0 -slope (a longitude of $K$ ) on $\partial M_{K}$.

Dehn surgery generalizes nicely to manifolds $M$ with a torus boundary component $T \subset \partial M$, where $M$ may not be the complement of knot in $S^{3}$. Let $\alpha \subset T$ be a slope, then $\alpha$-Dehn filling of $M$ on $T$ is the space $M(\alpha)=M \cup_{\varphi} V$, where $\varphi: \partial V \rightarrow T$ sends the meridian of $V$ onto $\alpha$. One difference in this scenario is that there may be no canonical way to distinguish a longitude on $T$, however, $\Delta(\alpha, \beta)$ is still well-defined for any pair of slopes, $\alpha$ and $\beta$.

\subsection{Cable spaces}

Let $V$ be a solid torus, and let $J$ be a $(p, q)$-curve inside $V$ (see Figure 2). The cable space, $C(p, q)$, is the space formed by removing a regular neighborhood of $J$. Let $T_{1}=\partial V$ and $T_{0}=\partial N(J)$. There is a properly embedded annulus, $A$, connecting the two boundary components such that $A \cap T_{1}$ is a $p / q$-curve (in terms of the standard meridian and longitude on $V$ ) and $\gamma=A \cap T_{0}$ is a $p q / 1$-curve (see Figure 2). Let $\mu$ and $\lambda$ be some choice of meridian and longitude for $T_{0}$. Then the slope $\gamma$ is called the cabling slope for $C(p, q)$.

Let $A^{\prime}$ be a properly embedded annulus such that $A^{\prime} \cap T_{0}$ is two $p q / 1$-curves, parallel to each other and to $\gamma$ (see Figure 2). Now, let $C(p, q)(\alpha)$ denote $\alpha-D e h n$ filling on $T_{0}$. Then, if $\alpha=\gamma$, this filling has the effect of capping off $A^{\prime}$ to form a separating 2-sphere, $S$, and capping off one boundary component of $A$ to form a disk, $D$, which intersects $T_{1}$ in a $p / q$-curve. The result is that $C(p, q)(\gamma)=\left(S^{1} \times D^{2}\right) \# L(q, p)$. 

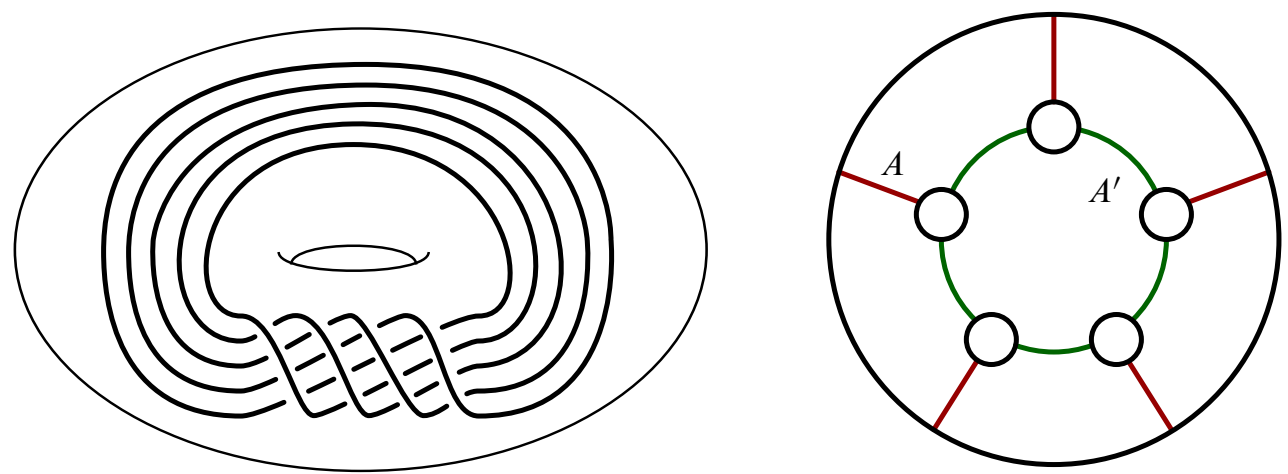

Figure 2: On the left, we see a $(4,5)$-curve $J$ inside a solid torus, $V$, and, on the right, we see a cross section of $V-N(J)$, along with two interesting annuli, $A$ and $A^{\prime}$.

Let $t: C(p, q) \rightarrow C(p, q)$ represent Dehn twisting along $A$. Then, we have that $\left.t^{l}(\mu)=\mu+l(p q \mu+\lambda)\right)=(l p q+1) \mu+l \lambda$. Since $C(p, q)(\mu)=S^{1} \times D^{2}$, it follows that $C(p, q)\left(t^{l}(\mu)\right)=S^{1} \times D^{2}$. So, slopes of the form $(l p q+1) / l$ all correspond to surgery slopes on $T_{0}$ that yield solid tori.

This shows that cable spaces have infinitely many fillings returning solid tori, all at distance one from the cabling slope.

On the other hand, we have the following lemma, which follows from the Cyclic Surgery Theorem (see Culler, Gordon, Luecke and Shalen [11]) and work of Gabai [17]; see Kang [26] for a proof and more general discussion.

Lemma 2.1 (a) Let $M \neq T^{2} \times I$ be an irreducible and $\partial$-irreducible 3-manifold with a torus boundary component, $T_{0}$. Let $T_{1}$ be an incompressible torus in $M$, distinct from $T_{0}$. If $\alpha$ and $\beta$ are slopes on $T_{0} \subset \partial M$ with $\Delta(\alpha, \beta) \geq 2$, such that $T_{1}$ is compressible in $M(\alpha)$ and $M(\beta)$, then $M$ is a cable space with cabling slope $\gamma$ such that $\Delta(\alpha, \gamma)=\Delta(\beta, \gamma)=1$.

(b) Let $M \neq T^{2} \times I$ be an irreducible and $\partial$-irreducible 3-manifold with a torus boundary component, $T_{0}$. Let $T_{1}$ be an incompressible torus in $M$, distinct from $T_{0}$. If $\alpha$ and $\beta$ are slopes on $T_{0} \subset \partial M$ with $\Delta(\alpha, \beta)=1$, such that $T_{1}$ is compressible in $M(\alpha)$ and $M(\beta)$, then either

(i) $M$ is a cable space with cabling slope $\alpha$ or $\beta$,

(ii) $\quad M$ is the exterior of a braid in a solid torus, $M(\alpha)$ and $M(\beta)$ are solid tori, and $\Delta\left(\eta_{\alpha}, \eta_{\beta}\right) \geq 4$, where $\eta_{\alpha}$ and $\eta_{\beta}$ are the induced slopes of the meridian on $T_{0}$. 


\subsection{Seifert fibered spaces}

A fibered solid torus is formed by gluing the ends of $D^{2} \times I$ together with a twist $\rho$ through $(2 \pi p) / q$, where $q \geq 1$ and $p$ and $q$ are relatively prime. There are two types of fibers: the central fiber, ie, the image of $(0,0) \times I$ after gluing, and the union of the $\operatorname{arcs} x \times I, \rho(x) \times I, \ldots, \rho^{q-1}(x) \times I$, for $x \neq(0,0)$.

A Seifert fibered space is a 3-manifold that can be decomposed as a disjoint union of circles (called fibers), where each fiber has a regular neighborhood homeomorphic to a fibered solid torus, ie, the fiber becomes the central fiber of the fibered solid torus. Viewing the neighborhood this way, if $q=1$, we say the fiber is ordinary. If $q \geq 2$, we say the fiber is exceptional with multiplicity $q$. In the latter case, the fibers surrounding the central fiber are called $(p, q)$-curves.

If $M$ is a Seifert fibered space, there is a natural projection $\pi: M \rightarrow \Sigma$ that identifies each fiber to a point. The surface $\Sigma$ is called the base space. We can record the exceptional fiber information in the form of cone points on $\Sigma$, so $M$ is a circle bundle over the resulting orbifold. Another way to recover $M$ is to remove a disk neighborhood of each cone point on $\Sigma$ and cross the resulting surface with $S^{1}$. The result is a manifold with torus boundary components. If we choose meridian and longitude coordinates for each boundary component so that the projection of the meridians to the base surfaces is one-to-one onto the boundary of the removed disks and the longitude is $\star \times S^{1}$ in the circle product, then $M$ is the result of Dehn filling on the boundary components along the slopes $p^{\prime} / q$, where $p p^{\prime} \equiv 1(\bmod q)$. If $M$ is a Seifert fibered space with base space $\Sigma$ and $n$ exceptional fibers with fibered solid torus neighborhoods consisting of $\left(p_{i} / q_{i}\right)$-curves for $i=1,2, \ldots, n$, we write $M=\Sigma\left(p_{1}^{\prime} / q_{1}, \ldots, p_{n}^{\prime} / q_{n}\right)$, or sometimes $M=\Sigma\left(q_{1}, \ldots, q_{n}\right)$. In fact, the homeomorphism type of $M$ is determined by $\Sigma$ and the Seifert invariants: $\left\{p_{1}^{\prime} / q_{1}, \ldots, p_{n}^{\prime} / q_{n}\right\}$, up to permutation, and up to the relation $\left\{p_{1}^{\prime} / q_{1}, p_{2}^{\prime} / q_{2}, \ldots, p_{n}^{\prime} / q_{n}\right\}=\left\{p_{1}^{\prime} / q_{1} \pm 1, p_{2}^{\prime} / q_{2} \mp 1, \ldots, p_{n}^{\prime} / q_{n}\right\}$. In other words, $\sum_{i=1}^{n} p_{i}^{\prime} / q_{i}$ is an invariant of $M$. Because of this, it is often useful to standardize the notation so that the Seifert invariants are all positive and less than one. To do this, we subtract out the integer part of each fraction and collect them in a single term, $b$. We write $M=\Sigma\left(b ; p_{1}^{\prime} / q_{1}, \ldots, p_{n}^{\prime} / q_{n}\right)$, where $0<p_{i}^{\prime}<q_{i}$ and $b \in \mathbb{Z}$.

A Seifert fibered space is called small if the base space is a sphere and the number of exceptional fibers is at most three.

Next, we recall a fact about Dehn filling on Seifert fibered manifolds that will be useful throughout this paper. Let $M$ be a Seifert fibered manifold with a torus boundary component $T \subset \partial M$. The fibering of $M$ induces a fibering of $T$, and the slope, $\gamma$, of the induced fibers on $T$ is called the Seifert slope of $T$. Now, the Seifert fibering 
of $M$ will extend to a Seifert fibering of $\alpha$-Dehn filling on $M$ provided that $\alpha \neq \gamma$. In fact, we have the following; see Heil [21] for a complete treatment of Dehn filling on Seifert fibered spaces with boundary.

Lemma 2.2 If $M$ is a Seifert fibered manifold with base surface $\Sigma$ and $n$ exceptional fibers, $T \subset \partial M$ is a torus boundary component (corresponding to a circle boundary component $C \subset \partial \Sigma$ ), and $\gamma$ is the Seifert slope $T$, then let $M(\alpha)$ denote $\alpha-D e h n$ filling on $T$, let $d=\Delta(\alpha, \gamma)$, and let $\widehat{\Sigma}=\Sigma \cup_{C} D^{2}$. Then,

(a) if $d \geq 2, M(\alpha)$ is a Seifert fibered space with base surface $\hat{\Sigma}$ and $n+1$ exceptional fibers (the original exceptional fibers, plus a new one of multiplicity $d$ ),

(b) if $d=1, M(\alpha)$ is a Seifert fibered space with base surface $\hat{\Sigma}$ and (the original) $n$ exceptional fibers,

(c) if $d=0, M(\alpha)=N \# L$, where $N$ is a Seifert fibered space with base surface $\hat{\Sigma}$ and (the original) $n$ exceptional fibers, and $L$ is a Lens space.

As an example, consider $D^{2}(a, b)$ with Seifert slope $r / s$, and let $d=\Delta(\mathrm{m} / \mathrm{l}, \mathrm{r} / \mathrm{s})=$ $|m s-l r|$. Then (as developed in [19]),

$$
D^{2}(a, b)(m / l)= \begin{cases}S^{2}(a, b, d) & \text { if } d \geq 2, \\ L\left(m, l b^{2}\right) & \text { if } d=1, \\ L(a, b) \# L(b, a) & \text { if } d=0 .\end{cases}
$$

\subsection{Montesinos knots}

A tangle is a pair $(B, A)$, where $B \cong B^{3}$ and $A$ is a pair of properly embedded arcs in $B$. A marked tangle is a tangle along with an identification of its boundary $\partial(B, A)=(S, S \cap A)$, which is a 2 -sphere with 4 distinguished points, with the pair $\left(S^{2},\{N E, N W, S W, S E\}\right)$. The trivial tangle is the tangle which is homeomorphic as a marked tangle to $\left(D^{2},\{2\right.$ points $\left.\}\right) \times I$. Let $h$ and $r$ be the tangle operations, where $h$ adds a positive horizontal half twist (right-handed), and $r$ is reflection in the $(N W / S E)$-plane.

Let $\left[c_{1}, c_{2}, \ldots, c_{m}\right]$ be a sequence of integers, and let

$$
p / q=\frac{1}{c_{1}+\frac{1}{c_{2}+\frac{1}{\cdots+c_{m}}}}
$$


Let $\mathcal{R}(1 / 0)$ denote the trivial tangle and form the rational tangle $\mathcal{R}(p / q)$ by applying the operation $\left(h^{c_{m}} r\right)\left(h^{c_{m-1}} r\right) \cdots\left(h^{c_{1}} r\right)$ to the trivial tangle. Note that, as an unmarked tangle, $\mathcal{R}(p / q)$ is trivial, one can just untwist it. On the other hand, Conway showed [9] that, as marked tangles, $\mathcal{R}(p / q)=\mathcal{R}\left(p^{\prime} / q^{\prime}\right)$ if and only if $p / q=p^{\prime} / q^{\prime}$.

A Montesinos link of length $n$ is a link formed by connecting $n$ rational tangles to each other in a standard fashion. We denote such a knot by $K\left[p_{1} / q_{1}, \ldots, p_{n} / q_{n}\right]$ (see Figure 3). In the special case where each $p_{i}= \pm 1$, we have what is called a pretzel knot. In this case, each tangle is just a strand of vertical twists, since $1 / q$ has the continued fraction expansion $[q]$. It is easy to see that Montesinos links of length one or two are the same. These links are called 2-bridge links, and will be denoted $K[p / q]$, where $p / q$ is the rational number describing the tangle twists.
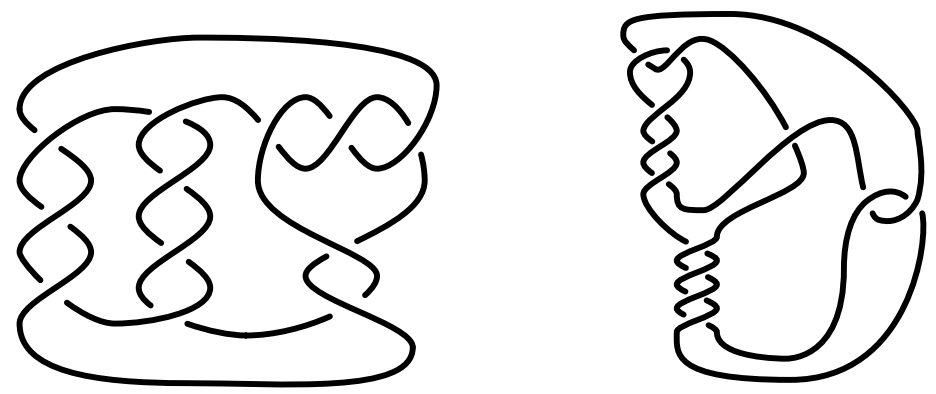

Figure 3: Above we have the Montesinos knot $K[1 / 3,1 / 4,-3 / 5]$ and the 2-bridge knot $K[43 / 95]$ (with continued fraction $[2,4,1,3,2]$ )

Montesinos links of length three are determined up to the same relations as Seifert fibered spaces, but when $n>3$, the cyclic order of the strands also matters. In either case, we can normalize the invariants and write $K\left[b ; p_{1} / q_{1}, \ldots, p_{n} / q_{n}\right]$ where $0<p_{i}<q_{i}$ and $b \in \mathbb{Z}$. In fact, we have the following proposition, which follows from Theorem 2.5 below.

Proposition 2.3 The branched double cover of $S^{3}$ along $K\left[p_{1} / q_{1}, \ldots, p_{n} / q_{n}\right]$ is the Seifert fibered space $S^{2}\left(p_{1} / q_{1}, \ldots, p_{n} / q_{n}\right)$.

We remark that it is often helpful to allow $p_{i} / q_{i}$ to be zero, $\infty$ or 1 for some $i$, in either the notation for Montesinos links or Seifert fibered spaces. For our purposes, this will only happen when the length $n$ is three or less, and the result should be clear from the context. For example, $K[1 / 3,-1 / 2,1 / 0]$ is the connected sum of a trefoil knot and a Hopf link, $K[1 / 3,2 / 7,0]=K[2 / 13]$, and $S^{2}(2,3,1)$ is a lens space. 


\subsection{Seifert fibered surgery on knots with symmetries}

In this section, we recall some known results about Seifert fibered surgery on knots that admit a strong inversion, have period two, or both. In what follows, let $K \subset S^{3}$ be a knot and let $\varphi: S^{3} \rightarrow S^{3}$ be a nontrivial orientation preserving involution such that $\varphi(K)=K$ and $C_{\varphi}=\operatorname{Fix}(\varphi) \neq \varnothing$. By the positive solution to the Smith conjecture, $C_{\varphi}$ is an unknotted circle in $S^{3}$; see Morgan and Bass [34].

Definition 2.4 If $C_{\varphi} \cap K \neq \varnothing$, then $\varphi$ is called a strong inversion of $K$ and $K$ is called strongly invertible. In this case, $C_{\varphi} \cap K=2$ points and $\varphi$ reverses the orientation of $K$.

If $C_{\varphi} \cap K=\varnothing$, then we say $\varphi$ is a cyclic symmetry of order 2 and that $K$ has period 2 .

In this paper, we will only be interested in strong inversions and cycles of period 2. For a more general treatment of Dehn surgery on knots with symmetries; see Motegi [36].
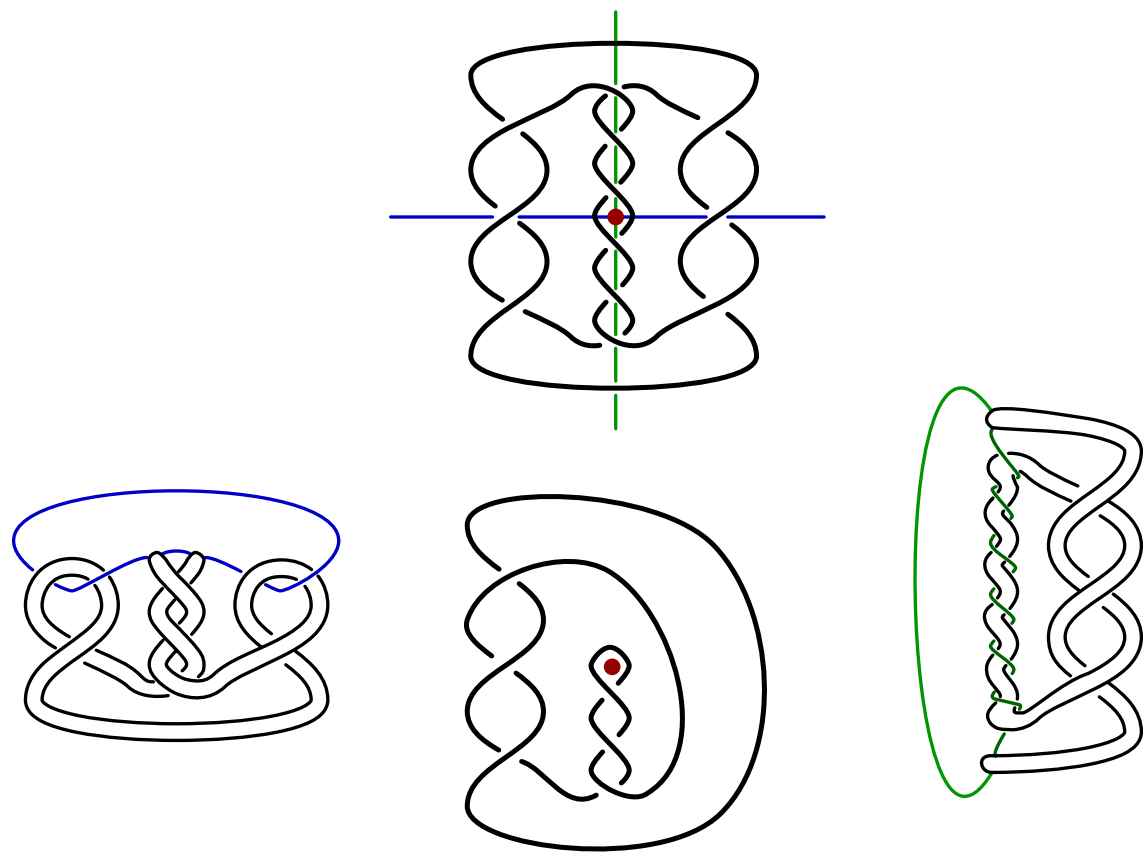

Figure 4: The knot $P(3,3,-6)$ with its three symmetries and the resulting quotients

First, let us consider strongly invertible knots. Let $K \subset S^{3}$ be a knot with a strong inversion $\varphi$. Then $\varphi$ restricts to an involution of the knot exterior, $M_{K}$, and the quotient of $M_{K}$ by the action of $\varphi$ is a tangle, $\mathcal{T}_{K}$. The well-known Montesinos trick 
gives a correspondence between Dehn filling on $M_{K}$ and rational tangle filling on $\mathcal{T}_{K}$. For details, see [19]. The following is originally due to Montesinos [33].

Theorem 2.5 Let $\mathcal{T}$ be a marked tangle. Then $\widetilde{\mathcal{T}}(r / s) \cong \widetilde{\mathcal{T}(-r / s)}$.

Let $L_{r / s}=\mathcal{T}_{K}(-r / s)$, so $L_{r / s}$ is a knot or a two-component link in $S^{3}$ with $K(r / s)$ as the double cover of $S^{3}$, branched along $L_{r / s}$. Suppose that $K(r / s)$ is a small Seifert fibered space. Let $\bar{\varphi}: K(r / s) \rightarrow K(r / s)$ be the involution induced by extending $\varphi$ across the filling solid torus. Then $K(r / s) / \bar{\varphi}=S^{3}$.

If $K$ is not a trefoil knot, then we can assume that $\bar{\varphi}$ is fiber-preserving [36]. Let $\pi: K(r / s) \rightarrow S^{2}$ be the Seifert fibration of $K(r / s)$. Let $C_{\bar{\varphi}}=\operatorname{Fix}(\bar{\varphi})$. If each component of $C_{\bar{\varphi}}$ is a fiber in $K(r / s)$, then $K(r / s) \backslash C_{\bar{\varphi}}$ admits a Seifert fibered structure. Since this structure is compatible with $\bar{\varphi}, S^{3} \backslash L_{r / s}$ admits a Seifert fibered structure. In other words, $L_{r / s}$ is a Seifert link.

Let $\hat{\varphi}: S^{2} \rightarrow S^{2}$ be the induced involution of the base orbifold. If one component of $C_{\bar{\varphi}}$ is not a fiber in $K(r / s)$, then $\hat{\varphi}$ is reflection across the equatorial circle, $C_{\hat{\varphi}}$, of $S^{2}$ and all of the cone points lie on $C_{\hat{\varphi}}$ [36]. In this case, $L_{r / s}=C_{\bar{\varphi}} / \bar{\varphi}$ is a length three Montesinos link; see Miyazaki and Motegi [32]. So, we have the following, as stated in [22].

Proposition 2.6 Let $K$ be a strongly invertible hyperbolic knot, and let $r / s \in \mathbb{Q}$. Let $L_{r / s}$ be the link obtained by applying the Montesinos trick to $K(r / s)$. If $K(r / s)$ is a small Seifert fibered space with base orbifold $S^{2}$, then $L_{r / s}$ is either a Seifert link or a Montesinos link.

Seifert links are well understood; see Burde and Murasugi [8] and Eisenbud and Neumann [14]. In the present paper, we will only be concerned with Seifert knots and Seifert links with two components, in which case we have the following.

Lemma 2.7 Let $L \subset S^{3}$ be a Seifert link with at most two components. Then $L$ is equivalent to one of the following:

(a) A torus knot.

(b) A two-component torus link.

(c) A two-component link consisting of a torus knot together with a core curve of the torus on which it lies. 
Note that, in particular, every component of a Seifert link is a torus knot or an unknot. Now let $K \subset S^{3}$ be a knot with a cycle symmetry $\varphi$ of order 2. Suppose $K(r / s)$ is a Seifert fibered space with base surface $S^{2}$, and let $\bar{\varphi}$ be the extension of $\left.\varphi\right|_{M_{K}}$ to $K(r / s)$. Then, $K(r / s)$ has a $\bar{\varphi}$-invariant Seifert fibered structure [32]. Let $C_{\bar{\varphi}}=\operatorname{Fix}(\bar{\varphi})$, and let $L_{r / s}=C_{\bar{\varphi}} / \bar{\varphi}$.

If $r$ is odd, then $L_{r / s}$ is a knot. If $r$ is even, then $L_{r / s}$ is a link. Let $K_{\varphi}=K / \varphi$. We call $K_{\varphi}$ the factor knot of $K$ (with respect to $\varphi$ ), and let $C_{\varphi}=\operatorname{Fix}(\varphi)$. In the case where $r$ is odd, we can view $L_{r / s}$ as the image of $C_{\varphi} / \varphi$ after $r / 2 s$ surgery on $K_{\varphi}$, so $L_{r / s}$ is a knot in $K_{\varphi}(r / 2 s)$. If $r$ is even, then $L_{r / s}$ is the image of $C_{\varphi} / \varphi$ in $K_{\varphi}(r / 2 s)$ together with the core of the surgery torus, so $L_{r / s}$ is a link in $K_{\varphi}(r / 2 s)$. Let $\pi: K(r / s) \rightarrow S^{2}$ be a Seifert fibering of $K(r / s)$, and let $\hat{\varphi}$ be the induced involution of $S^{2}$, with fixed point set $C_{\hat{\varphi}}$. In [32], it is shown that if $K$ is not a torus knot or a cable of a torus knot, then no component of $C_{\bar{\varphi}}$ is a fiber in $K(r / s)$ and $C_{\hat{\varphi}}$ is the equatorial circle in $S^{2}$. This implies that $\hat{\varphi}$ is reflection across the equator. Since $\bar{\varphi}$ is fiber preserving, $\hat{\varphi}$ must pair up cone points in the northern hemisphere with cone points in the southern hemisphere. Let $k$ denote the number of cone points in the northern hemisphere. For our purposes, $k=0$ or $k=1$. From [32], we have the following.

Lemma 2.8 (a) If $k=0$, then $K_{\varphi}(r / 2 s)=K(r / s) / \bar{\varphi} \cong S^{3}$.

(b) If $k=1$, then $K_{\varphi}(r / 2 s)=K(r / s) / \bar{\varphi}$ is a lens space.
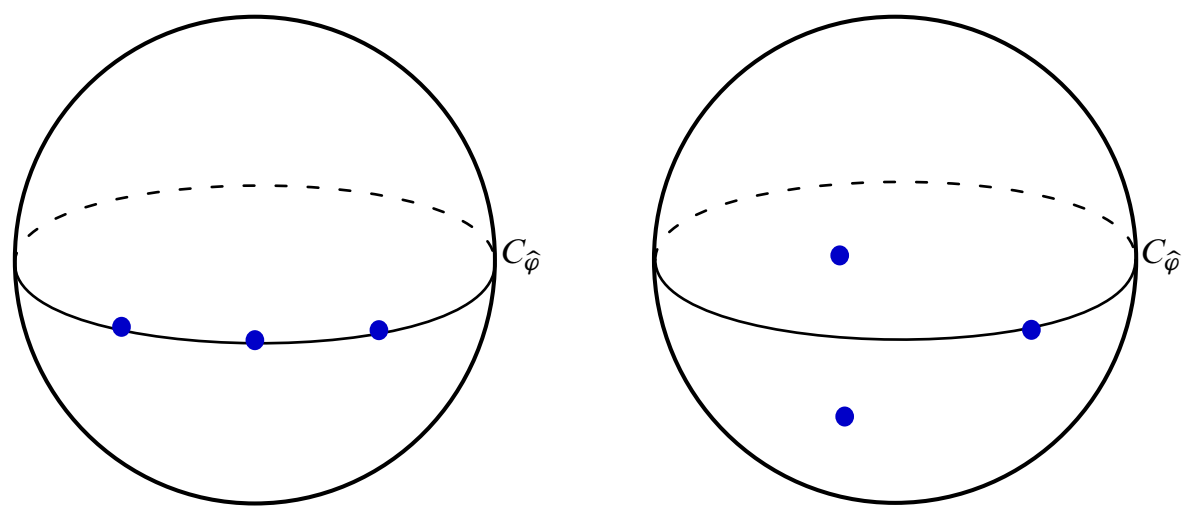

Figure 5: Possible configurations of cone points in the base sphere of a small Seifert fibered space

Note that $S^{3}$ and $S^{2} \times S^{1}$ are not lens spaces. These facts can be helpful in obstructing Seifert fibered surgeries, based on the knot type of $K_{\varphi}$. Throughout, $U$ will represent the unknot. 
Corollary 2.9 Let $K \subset S^{3}$ be a period 2 hyperbolic knot with factor knot $K_{\varphi}$. Suppose $K(r / s)$ is a small Seifert fibered space.

(a) If $K_{\varphi}=T_{p, q}$ and $r$ is even, then $\Delta(p q, r / 2 s)=1$, so $|r-2 s p q|=2$.

(b) If $K_{\varphi}=T_{p, q}$ and $r$ is odd, then $\Delta(p q, r / 2 s)=1$, so $|r-2 s p q|=1$.

(c) If $K_{\varphi}=U$ and $k=0$, then $|r| \leq 2$.

(d) If $K_{\varphi}=U$ and $k=1$, then $|r| \geq 3$.

Proof If $K(r / s)$ is a small Seifert fibered space, then $K_{\varphi}(r / 2 s)$ is a lens space if $k=1$ and is $S^{3}$ if $k=0$. Such surgeries on $U$ and $T_{p, q}$ are well understood (see [19]).

\subsection{Some exceptional Dehn surgery results}

There are many important results in the study of exceptional Dehn surgery that give limitations on which slopes can be exceptional for a hyperbolic knot $K$. Below, we present some of the results that will be used in this paper. First, we state an important result of Lackenby and Meyerhoff [27] that tells us that exceptional fillings are always "close" to each other.

Theorem 2.10 Suppose $M$ is a hyperbolic manifold with torus boundary component $T \subset \partial M$ and that $\alpha$ and $\beta$ are exceptional filling slopes on $T$. Then $\Delta(\alpha, \beta) \leq 8$.

The distance bound of 8 above can be improved if one specifies the type of space for each of $M(\alpha)$ and $M(\beta)$. Let $S$ and $T$ represent the sets of reducible and toroidal manifolds, respectively. Let $L$ represent the set of lens spaces. Let $\Delta\left(C_{1}, C_{2}\right)$ represent the largest possible value of $\Delta(\alpha, \beta)$ such that there exists a hyperbolic manifold $M$ with $M(\alpha)$ a manifold of type $C_{1}$ and $M(\beta)$ a manifold of type $C_{2}$ (we will always consider manifolds with one boundary component, though the theory is more general). The following table presents the known values of $\Delta\left(C_{1}, C_{2}\right)$.

\begin{tabular}{c|c|c|c|c} 
& $S$ & $T$ & $S^{3}$ & $L$ \\
\hline$S$ & 1 & 3 & $?$ & 1 \\
\hline$T$ & & 8 & 2 & $?$ \\
\hline$S^{3}$ & & & 0 & 1 \\
\hline$L$ & & & & 1
\end{tabular}

Notice that in the case of $\left(S, S^{3}\right)$, this is equivalent to the cabling conjecture, and in the case of $(T, L)$, the bound is known to be either 3 or 4; see Lee [28]. For a 
more thorough discussion of these bounds, the manifolds achieving them, and precise references, see Gordon [18; 19].

Suspiciously absent from the above table are bounds on the distance between a (nonlens space) small Seifert fibered surgery and the other types of exceptional surgeries. These seem to be the most difficult cases to analyze, and, in particular, it is not known whether or not the distance 8 bound of Lackenby and Meyerhoff can be improved in most of the cases (though, see Theorems 2.11 and 2.12 below).

The following is a consequence of Boyer, Gordon and Zhang [5, Corollary 7.6, Proposition 14.1 and Proposition 16.1].

Theorem 2.11 For any hyperbolic manifold $M$, if $M(\alpha)=A \cup_{T^{2}} B$ is toroidal with one of $A$ or $B$ non-Seifert fibered, then for any slope $\beta$ such that $M(\beta)$ is a Seifert fibered space, $\Delta(\alpha, \beta) \leq 6$.

Since many of the pretzel knots studied below are genus one, it will be helpful for us to have the following result, which gives particularly strong bounds on small Seifert fibered surgery slopes of such knots; see Boyer, Gordon and Zhang [4].

Theorem 2.12 Let $K$ be a hyperbolic knot of genus one such that $K(0)$ is a nonSeifert fibered toroidal manifold. If $K(\alpha)$ is a small Seifert fibered space for some $\alpha \in \mathbb{Q}$, then $\Delta(\alpha, 0) \leq 3$.

\subsection{Montesinos links, torus links and invariants from knot theory}

In this section we give a very brief overview of some knot and link invariants and how they will be used to obstruct the quotient links encountered in this paper from being Seifert links or Montesinos links. We will present a series of criteria that will applied in each of the following sections.

A link is called $k$-almost alternating if it has a $k$-almost alternating diagram, but no $(k-1)$-almost alternating diagram, ie, if it has a diagram $D$ such that changing $k$ crossings of $D$ gives a new diagram that is alternating, but no such diagram where the same result is achieved after $k-1$ crossing changes.

Recall that the Khovanov homology, $\mathrm{Kh}(L)$, is a bigraded abelian group associated to $L$, and that the width of $\mathrm{Kh}(L)$ is the number of diagonals that support a nontrivial element in $\operatorname{Kh}(L)$. Denote this width by $|\operatorname{Kh}(L)|$. Then we have the following theorem (see, for example, Asaeda and Przytycki [2]).

Theorem 2.13 Let $L$ be a nonsplit $k$-almost alternating link. Then $|\operatorname{Kh}(L)| \leq k+2$. 
It has been shown by Abe and Kishimoto [1] that any Montesinos link is either alternating or 1-almost-alternating, so we have our first obstruction criterion.

\section{Criterion 2.14 If $|\operatorname{Kh}(L)| \geq 4$, then $L$ is not a length-three Montesinos link.}

When we encounter links $L$ that do not satisfy this criterion, then we will use the following program to show they are not a Montesinos link. We will generate a list of all Montesinos links whose crossing numbers are compatible with that of $L$ (ie, less than the number of crossings in a diagram of $L$ ). (Note that the crossing number of a Montesinos link is well understood; see Lickorish and Thistlethwaite [30].) We will then check this list for elements that, if $L$ is a knot, have the same determinant, Alexander polynomial, Jones polynomial, Khovanov homology, and, if need be, Kauffman polynomial or HOMFLYPT polynomial, and that, if $L$ is a 2 -component link, have the same determinant, Jones polynomial, Khovanov homology, and if need be, Kauffman polynomial or HOMFLYPT polynomial. We will refer to this method as Method 1 . This very large number of computations was performed using the KnotTheory package for Mathematica ${ }^{\circledR}$ [43].

Examples of the Mathematica files used to implement Method 1 and to calculate knot invariants throughout this paper are available on the author's webpage, and further information will be provided upon request.

Next, we observe that if $K$ is a length three Montesinos link, then it is the union of 2-bridge knots and unknots. If $K$ is the union of two unknots, then it has the form $K\left[p_{1} / q_{1}, p_{2} / q_{2}, p_{3} / q_{3}\right]$, where each $p_{i}$ is even. If one component of $K$ is the 2-bridge knot $K[p / q]$, then $K$ has the form $K\left[p_{1} / q_{1}, p_{2} / q_{2}, x / q\right]$ with $q_{1}$ and $q_{2}$ even and with $x=p$ or $\bar{p}$, where $p \bar{p} \equiv 1(\bmod q)$. If we consider the unknot a 2-bridge knot, then we have the following criterion.

Criterion 2.15 If $K$ is a 2-component link such that one component is not a 2-bridge knot, then $K$ is not a Montesinos link.

Using Method 1 and Criteria 2.14 and 2.15, any knot or link we encounter that we claim is not a Montesinos knot or link is shown to not be a Montesinos knot or link.

Now we recall some facts about torus knots (see, for example, Cromwell [10]). Let $T(p, q)$ be the $(p, q)$-torus link for $p>q \geq 2$, where $T(p, q)$ is a knot if and only if $p$ and $q$ are coprime. Then, $T(p, q)$ is a positive link, ie, has a diagram with all positive crossings. Furthermore, in the case of a torus knot, $2 g(T(p, q))=(p-1)(q-1)$, where $g(K)$ denotes the genus of the knot $K$, and $\operatorname{det}(T(p, q))=p$ if $q$ is even, and 1 if both $p$ and $q$ are odd, where $\operatorname{det}(L)$ denotes the determinant of the link $L$. 
Let $s(K)$ denote the Rasmussen invariant of $K$, as defined in [41], where the following was shown.

Proposition 2.16 If $K$ is a positive knot, then $s(K)=2 g(K)$.

Recall that $2 g(K)$ is bounded below by the breadth of the Alexander polynomial, which we denote $\operatorname{br}\left(\Delta_{K}(t)\right)$. This gives us the following criterion.

Criterion 2.17 If $s(K)<\operatorname{br}\left(\Delta_{K}(t)\right)$ or $2 g(K) \neq s(K)$, then $K$ is not a torus knot.

We also have, by our discussion above, the following.

Criterion 2.18 If $\operatorname{det}(K)>s(K)+1$, then $K$ is not a torus knot.

If we consider the unknot a torus knot, then each component of a two component Seifert link is a torus knot, so we have the following criterion.

Criterion 2.19 If $L$ is a two-component link such that a component is not a torus knot, then $K$ is not a Seifert link.

In what follows, Criteria 2.17, 2.18 and 2.19 often suffice to prove that a link is not a Seifert link. In the few cases where they fail, further argument is given to accomplish the feat.

\section{The case of $\left(2,\left|q_{2}\right|,\left|q_{3}\right|\right)$}

Let $K_{p, q}$ be the hyperbolic pretzel knot $P(-2,2 p+1,2 q+1)$ (see Figure 6). Since Ichihara and Jong have shown that $K_{p, p}$ admits no small Seifert fibered surgery [24], and by interchanging $p$ and $q$ if necessary, we may assume that $|q|>|p|$ if $p$ and $q$ have the same sign and $p>0$ otherwise. Let $\alpha_{r}=4(p+q+1)-r$, for $r \in \mathbb{Q}$.

Note that $\alpha_{r}$ is chosen this way so that $\alpha_{r}$-surgery on $K_{p, q}$ will correspond with $r$-filling of $\mathcal{T}_{p, q}$. This becomes clear if one carefully follows through the process of obtaining $\mathcal{T}_{p, q}$ from $K_{p, q}$ by applying the Montesinos trick and isotoping.

Our first result is the following.

Theorem 1.1 The hyperbolic pretzel knot $P(-2,2 p+1,2 q+1)$, with the conventions discussed above, admits a small Seifert fibered surgery if and only if $p=1$, in which case it admits precisely the following small Seifert fibered surgeries:

- $P(-2,3,2 q+1)(4 q+6)=S^{2}(1 / 2,-1 / 4,2 /(2 q-5))$

- $P(-2,3,2 q+1)(4 q+7)=S^{2}(2 / 3,-2 / 5,1 /(q-2))$ 

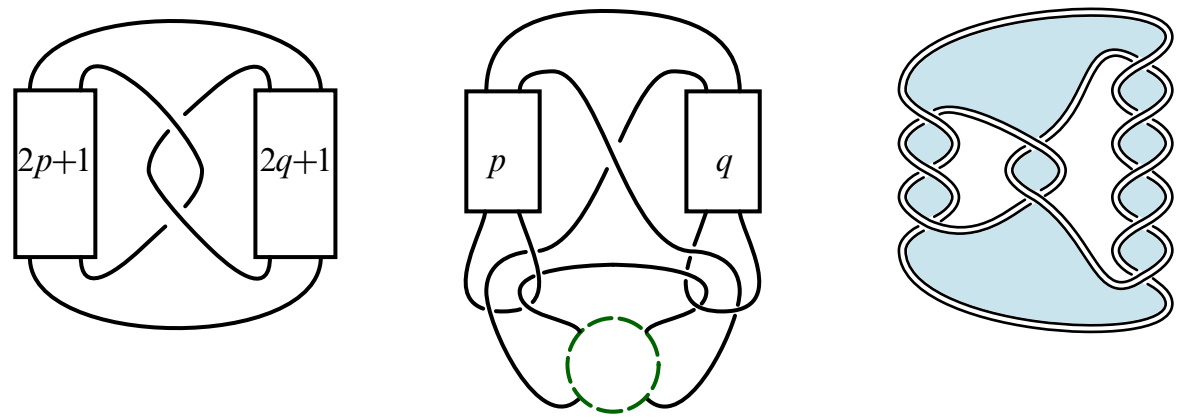

Figure 6: The pretzel knot $P(-2,2 p+1,2 q+1)$, the quotient tangle $\mathcal{T}_{p, q}$ and the pretzel knot $P(-2,5,-3)$, shown as the boundary of a punctured Klein bottle

We remark that the existence of these exceptional surgeries was previously known; see Eudave-Muñoz [15].

The key fact in our method of analyzing these knots is that they are strongly invertible. Let $\mathcal{T}_{p, q}$ be the tangle obtained by performing the Montesinos trick (see Figure 6). We now have the advantage of viewing the surgery space $K_{p, q}\left(\alpha_{r}\right)$ as the branched double cover of $S^{3}$ along $\mathcal{T}_{p, q}(r)$. It is easy to verify the two classes of exceptional surgeries in Theorem 1.1 by noticing that $\mathcal{T}_{1, q}(1)$ and $\mathcal{T}_{1, q}(2)$ are the Montesinos links $K[2 / 3,-2 / 5,1 /(q-2)]$ and $K[1 / 2,-1 / 4,2 /(2 q-5)]$, respectively; see Figure 7 .

The proof that the $K_{p, q}$ admits no other small Seifert fibered surgeries is accomplished by the following two lemmas and the techniques of Section 2.7.

Lemma 3.1 If $K_{p, q}\left(\alpha_{r}\right)$ is a small Seifert fibered space for $p \neq 1$, then $|p| \leq 8$ and $|q| \leq 8$.

Lemma 3.2 If $K_{1, q}\left(\alpha_{r}\right)$ is a small Seifert fibered space for $r \notin\{1,2\}$, then $|q| \leq 8$.

Before we prove these lemmas, we should remark on the possible surgery slopes $\alpha_{r}$. We notice that each knot $K_{p, q}$ bounds a punctured Klein bottle at slope $\alpha_{0}$ (see Figure 6). It follows that $K_{p, q}\left(\alpha_{0}\right)$ is toroidal. By Theorem 2.10, it follows that if $K_{p, q}\left(\alpha_{r}\right)$ is a small Seifert fibered space, then $\Delta\left(\alpha_{r}, \alpha_{0}\right) \leq 8$.

In many cases, it should be possible to reduce this distance bound to 5, but this is dependent on work in progress by Boyer, Gordon and Zhang [5]. However, using Theorem 2.11, we can fairly easily show the following lemma.

Lemma 3.3 If $K_{p, q}\left(\alpha_{r}\right)$ is a small Seifert fibered space, and $|p|,|q| \geq 4$, then $\Delta\left(\alpha_{r}, \alpha_{0}\right) \leq 6$. 

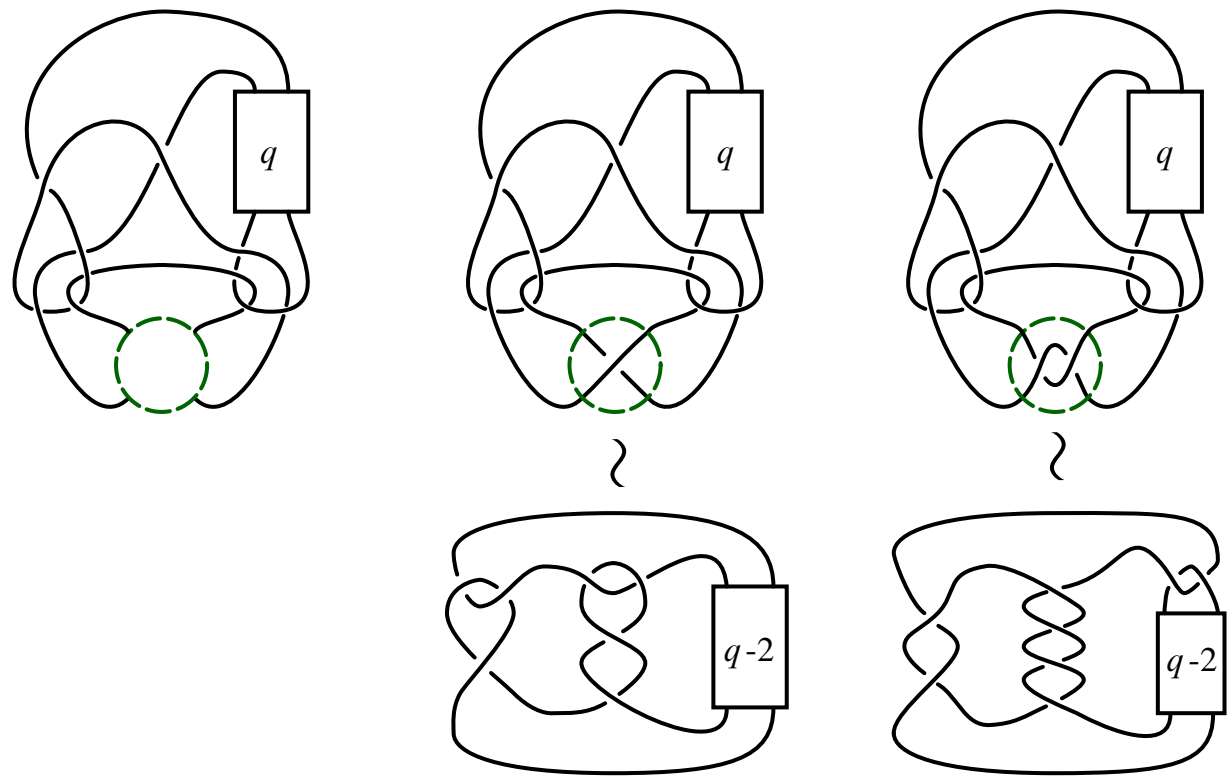

Figure 7: The tangle $\mathcal{T}_{1, q}$, along with fillings $\mathcal{T}_{1, q}(1)$ and $\mathcal{T}_{1, q}(2)$ and the respective Montesinos links that result after isotopy: $K[2 / 3,-2 / 5,1 /(q-2)]$ and $K[1 / 2,-1 / 4,2 /(2 q-5)]$

Of course, if $r$ is integral, this means that $|r| \leq 6$, and if we have $r / s \in \mathbb{Q}$, we have that $|4(p+q+1) s-r| \leq 6$.

Proof of Lemma 3.3 We begin by noticing that $K_{p, q}\left(\alpha_{0}\right)=D^{2}(2,2) \cup_{T^{2}} X_{p, q}$ (see Figure 8). Under the hypotheses of the lemma, we will show that $X_{p, q}$ is not a Seifert fibered space, so Theorem 2.11 gives us the desired bound. If $p=1$, then $X_{1, q}=D^{2}(3,|q-1|)$, and Theorem 2.11 does not apply.

Consider the following fillings on $X_{p, q}$ (see Figure 9):

$$
\begin{aligned}
X_{p, q}(0) & =L(p+q+1,1) \\
X_{p, q}(\infty) & =L(4 p q-2 p-2 q-3,2 p q-q-2) \\
X_{p, q}(-1) & =S^{2}(1 / 3,1 /(p-1), q /(q-1))
\end{aligned}
$$

If $X_{p, q}$ is a Seifert fibered space, then it has, for its base surface, either $D^{2}$ or $M^{2}$ (the Möbius band). We will make use of Lemma 2.2. If $X_{p, q}$ is Seifert fibered over the disk with more than two exceptional fibers it cannot have lens space fillings. If $X_{p, q}$ has the form of $D^{2}(a)$, then it cannot have fillings with three exceptional fibers, so $X_{p, q}(-1)$ must be a lens space. This implies that $p=2$ or $q=2$. If $X_{p, q}$ has base surface $M^{2}$, 

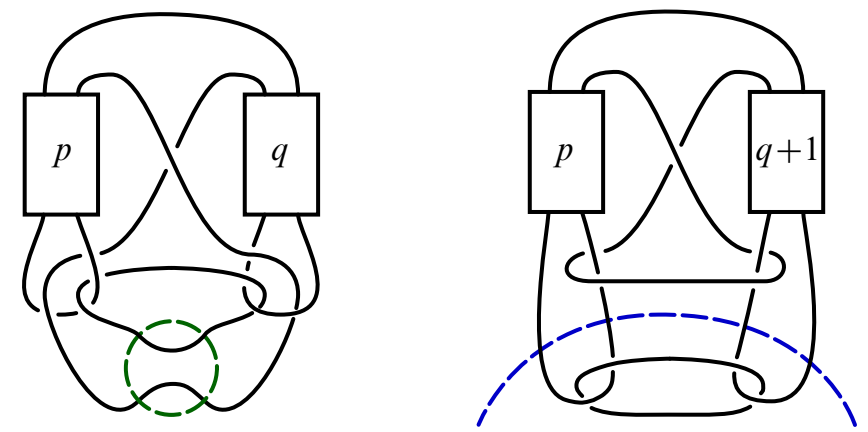

Figure 8: The link $\mathcal{T}_{p, q}(0)$, whose branched double cover corresponds to $\alpha_{0}$-surgery on $K_{p, q}$

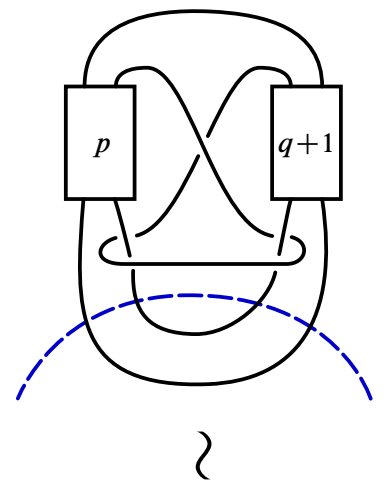

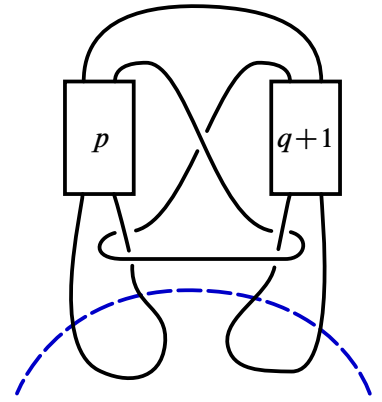

2

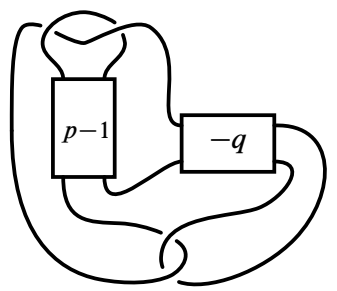

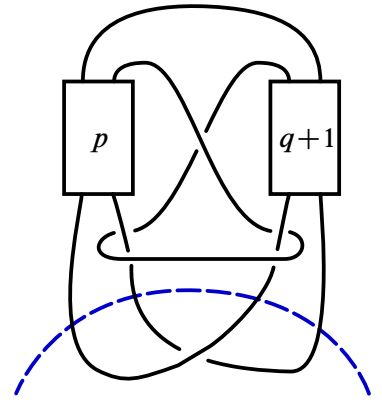

2

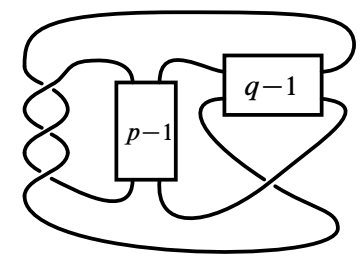

Figure 9: The three fillings, $0, \infty$ and 1 , on $\mathcal{T}_{p, q}$ used to show that $X_{p, q}$ is not Seifert fibered

then it can only have lens space fillings or fillings with at least three exceptional fibers, two of which have multiplicity two. Thus, we must have $p, q=2,3$. So, assume $X_{p, q}=D^{2}(a, b)$.

In this case, $X_{p, q}$ has one reducible filling at slope $\gamma$ and the property that any lens space filling must be at distance one from $\gamma$. By considering the three fillings given above, it follows that $\gamma=0, \infty$ or \pm 1 . If $\gamma=-1$, then $X_{p, q}(-1)$ must be reducible, 
so $p=1$ or $q=1$, both of which are not allowed values. If $\gamma=0$ or if $\gamma=\infty$, then $X_{p, q}(-1)$ must be a lens space, so $p=2$ or $q=2$. Finally, if $\gamma=1$, then the filling $X_{p, q}(-1)$ is at distance two from the reducible filling, so it must have an exceptional fiber of multiplicity 2 . It follows that $p=3$ or $q=3$.

We remark that the lemma could be strengthened to say that $X_{p, q}$ is non-Seifert fibered if and only if $p \neq 1$ by showing that $X_{p, q}(1)$ is neither reducible, a lens space, or a small Seifert fibered space with finite fundamental group, as would need to be the case given the different Seifert fibered structures $X_{p, q}$ might have. However, we will not need anything stronger than what we have proved.

Proof of Lemma 3.1 Suppose that $p \neq 1$ and remove a ball around the $q$-twists of $\mathcal{T}_{p, q}(r)$ to form the tangle $\mathcal{S}_{p, r}$ (see Figure 10). Let $N_{p, r}$ denote the branched double cover of $\mathcal{S}_{p, r}$. First, we will show that $N_{p, r}$ is hyperbolic.

We begin by showing some interesting fillings of $N_{p, r}$ (see Figure 10):

$$
\begin{aligned}
N_{p, r}(-1 / q) & =K_{p, q}\left(\alpha_{r}\right) \\
N_{p, r}(0) & =D^{2}(1 / 2,-1 / 2) \cup_{T^{2}} D^{2}(-1 / 2, p /(2 p-1)) \\
N_{p, r}(\infty) & =T(2,2 p+3)\left(\alpha_{r}\right)=S^{2}(-1 / 2,-1 /(r+2),-2 /(2 p+3)) \\
N_{p, r}(-1) & =K[-2 p /(6 p+1)]\left(\alpha_{r}\right)
\end{aligned}
$$

We remark $N_{p, r}(\infty)$ and $N_{p, r}(-1)$ correspond to $\left(\alpha_{r}\right)$-surgery on $K_{p, 0}$ and $K_{p,-1}$, respectively. The latter is a 2 -bridge knot with no exceptional fillings (if $p \neq 1$ ), according to the classification by Brittenham and $\mathrm{Wu}([7])$. Thus, $N_{p, r}(-1)$ is hyperbolic if $p \neq 1$.

Now, suppose that $N_{p, r}$ is not hyperbolic, so it must be reducible, $\partial$-reducible, Seifert fibered, or toroidal by geometrization. However, $N_{p, r}$ cannot be reducible, since it has two distinct irreducible fillings at slopes 0 and -1 (this follows from the solution to the knot complement problem by Gordon and Luecke [20]). Similarly, it cannot be Seifert fibered, since it has a hyperbolic filling at slope -1 . It follows that $N_{p, r}$ cannot be $\partial$-reducible, since the only irreducible, $\partial$-reducible manifold with torus boundary is Seifert fibered, namely, the solid torus.

Finally, suppose that $N_{p, r}$ is toroidal. If any essential torus were nonseparating, then all fillings of $N_{p, r}$ would contain an essential nonseparating torus, which is false here. Suppose any essential torus is separating, and decompose $N_{p, r}$ along an outermost such torus, $F$, so that $N_{p, r}=A \cup_{F} B$ with $A$ atoroidal and $\partial N_{p, r} \subset B$. If we assume, for a contradiction, that $N_{p, r}(-1 / q)$ is small Seifert fibered for some $q$ with $|q|>8$, then we have that $F$ compresses in $B(-1), B(\infty)$, and $B(-1 / q)$. It follows, from 

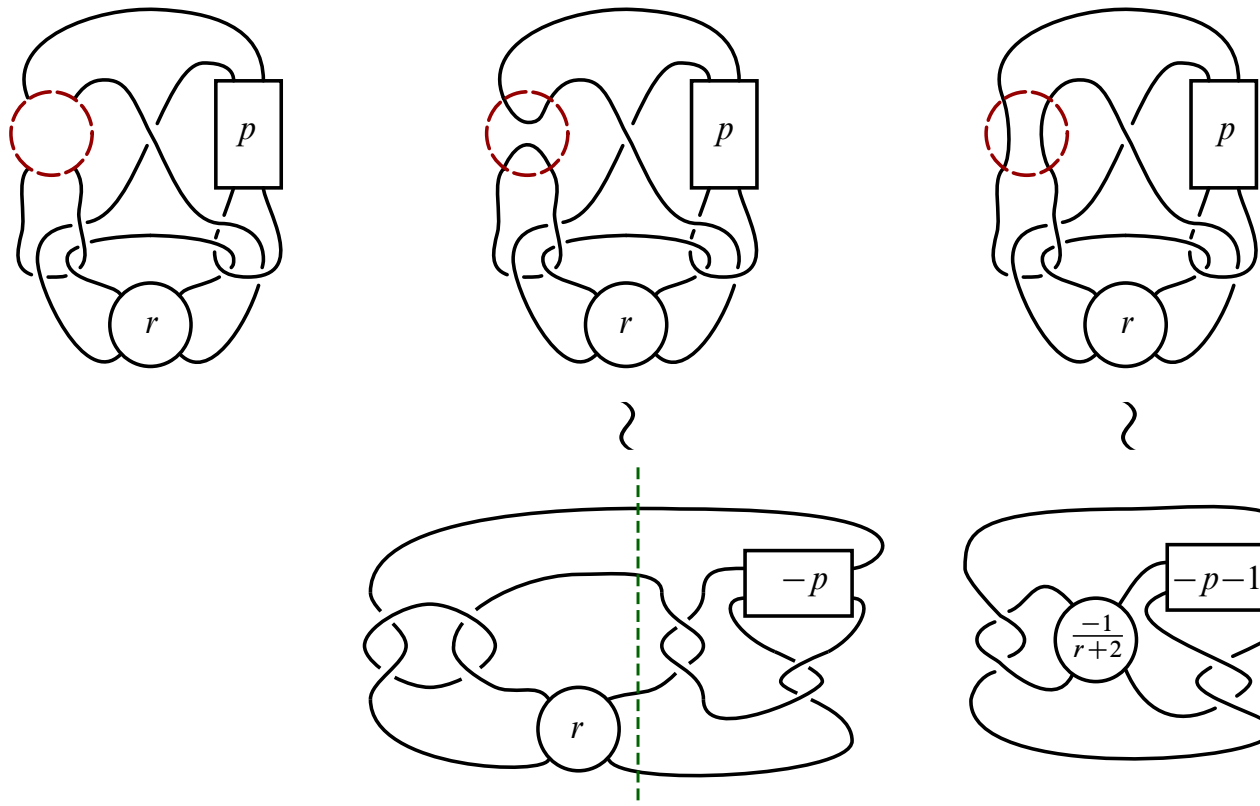

2

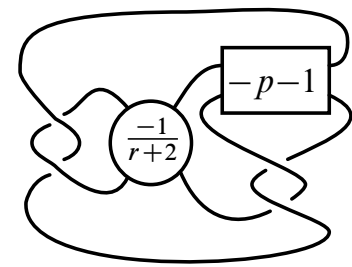

Figure 10: The tangle $\mathcal{S}_{p, r}$, along with two fillings, $\mathcal{S}_{p, r}(0)$ and $\mathcal{S}_{p, r}(\infty)$, and their equivalents after isotopy

Lemma 2.1 that $B$ is a cable space with cabling slope $\gamma=0$. But $N_{p, r}(0)$ is neither reducible nor a lens space, so we reach a contradiction. It follows that $N_{p, r}$ is not toroidal, and must be hyperbolic.

Since $N_{p, r}$ is hyperbolic, and $N_{p, r}(\infty)$ is exceptional, it follows that for any exceptional filling $N_{p, r}(-1 / q), \Delta(\infty,-1 / q) \leq 8$, by Theorem 2.10. It follows that $|q| \leq 8$, as desired. A similar argument shows that $|p| \leq 8$, as well.

Proof of Lemma 3.2 We will proceed as in the lemma above by analyzing the tangle $\mathcal{S}_{r}=\mathcal{S}_{1, r}$ formed by removing a ball containing the $q$-twist region of knot $\mathcal{T}_{1, q}(r)$. We will show that the branched double cover $N_{r}$ of $\mathcal{S}_{r}$ is hyperbolic.

Consider the following fillings on $N_{r}$ (see Figure 11). Assume for a contradiction that $|q| \geq 9$ and $K_{p, q}\left(\alpha_{r}\right)=N_{r}(-1 / q)$ is a small Seifert fibered space. Then

$$
\begin{aligned}
N_{r}(-1 / q) & =K_{p, q}\left(\alpha_{r}\right), \\
N_{r}(0) & =S^{2}(1 / 2,-1 / 2,1 /(2-r)), \\
N_{r}(\infty) & =S^{2}(1 / 2,-2 / 5,-1 /(r+2)),
\end{aligned}
$$




$$
\begin{aligned}
N_{r}(-1) & =S^{2}(1 / 3,-1 / 4,-1 / r), \\
N_{r}(-1 / 2) & =S^{2}(-1 / 3,2 / 5,-1 /(r-1)), \\
N_{r}(1) & =K[-2 / 7]\left(\alpha_{r}\right) .
\end{aligned}
$$

It is clear from this that $N_{r}$ is irreducible (again, by [20]), since it has distinct irreducible
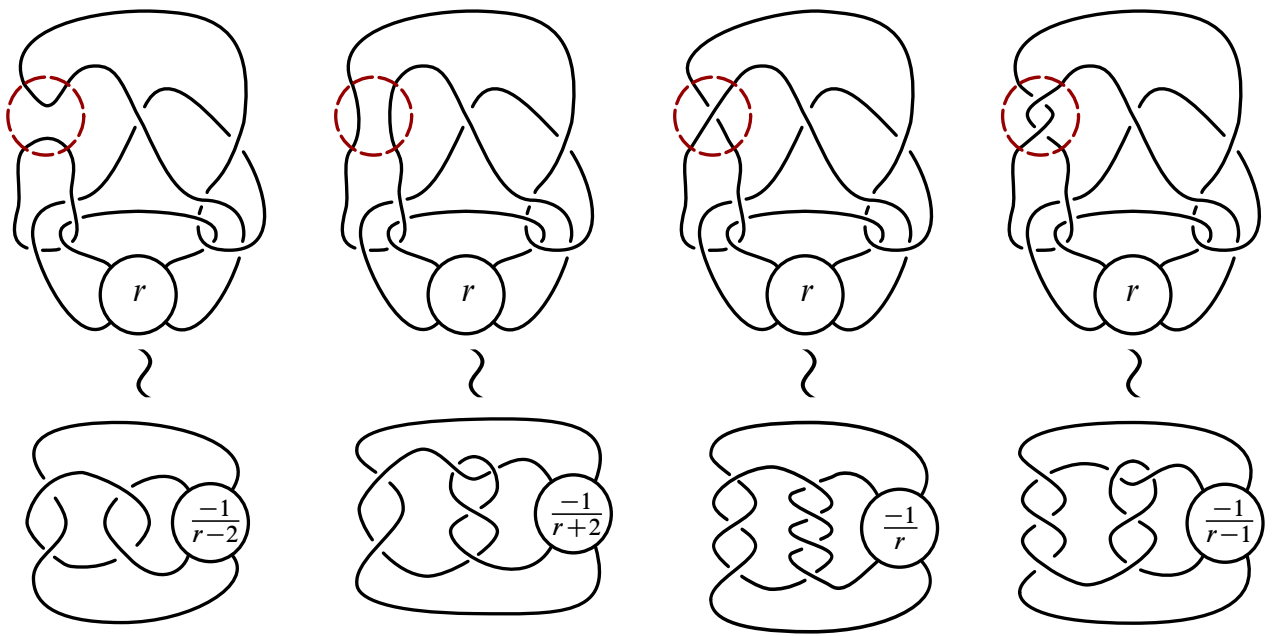

Figure 11: Four fillings, $0, \infty, 1$ and 1/2, of the tangle $\mathcal{S}_{r}$ that help to prove that $N_{r}$ is hyperbolic if $r \neq 0,1,2$

fillings, for any value of $r$. Suppose that $N_{r}$ is Seifert fibered. Since, for all values of $r, N_{r}$ has fillings that are Seifert fibered with base surface $S^{2}$, but do not contain a pair of exceptional fibers of multiplicity 2, the base surface of $N_{r}$ is orientable, ie, $D^{2}$. A Seifert fibered space with connected boundary with a small Seifert fibered filling must have 2 or 3 exceptional fibers. Furthermore, since no slope is distance one from $0, \infty$, and $-1, N_{r}$ has 2 exceptional fibers, ie, $N_{r}=D^{2}(a, b)$.

By the classification of exceptional surgeries on 2-bridge knots [7], $N_{r}(1)$ is exceptional if and only if $\alpha_{r} \in\{0,1,2,3,4\}$. In this case, $p=1$ and $q=-1$, so $\alpha_{r}=4-r$ and this is the equivalent to $r \in\{4,3,2,1,0\}$. However, we already know that if $r=0,1,2$, then $N_{r}(-1 / q)$ is exceptional, so we only need to consider $r=3,4$.

If $r=3$, then, by considering $N_{3}(\infty)$, we see that $a=5$, and by considering $N_{3}(-1)$, we see that $a$ cannot be 5 . If $r=4$, then by considering $N_{4}(-1 / 2)$, we see that $a=3$, and by considering $N_{4}(\infty)$, we see this is impossible. It follows that $N_{r}$ cannot be Seifert fibered if $r \notin\{0,1,2\}$.

It follows that $N_{r}$ is non-Seifert fibered and, thereby, $\partial$-irreducible. If $N_{r}$ were toroidal, since it has atoroidal fillings at distance two, it must be a cable space, by 
Lemma 2.1. However, the only cabling slope $\gamma$ that satisfies $\Delta(\gamma,-1 / 2)=1$ and $\Delta(\gamma, \infty)=1$ is $\gamma=0$, in which case we must have $N_{r}(0)$ be reducible or a lens space. So, we must have $r=3$ and $N_{3}(0)$ is a lens space. Suppose $N_{3}=A \cup_{T^{2}} B$ with $A$ atoroidal and $\partial N_{3} \subset B$.

Then, because $B(\alpha)=S^{1} \times D^{2}$ for $\alpha \in\{-1 / q, \infty,-1,-1 / 2,1\}$ each of these fillings induces a filling $\eta_{\alpha}$ on $A$. Since $B(0)=\left(S^{1} \times D^{2}\right) \# L$ for some lens space $L$, and since $N_{3}(0)$ is a lens space, it follows that $A\left(\eta_{0}\right)=S^{3}$, so $A$ is a knot complement. By construction, $A$ is atoroidal. If $A$ were Seifert fibered, then, by considering $A\left(\eta_{\infty}\right)=N_{3}(\infty)$ and $A\left(\eta_{-1}\right)=N_{3}(-1)$ just as before, we reach a contradiction.

It follows that $N_{r}$ must be hyperbolic (for $r \notin\{0,1,2\}$ ). An application of Theorem 2.10 gives us that $\Delta(-1 / q, \infty) \leq 8$ if $N_{r}(-1 / q)$ is a small Seifert fibered space, which proves the lemma.

\subsection{Completing the proof of Theorem 1.1}

The work above leaves us with a finite list of knots and links $L_{p, q, r}=\mathcal{T}_{p, q}(r)$ whose branched double covers might be Seifert fibered. We must consider nonintegral $r$ if and only if $p$ and $q$ are both positive. In the event of a nonintegral slope $r / s$, we may assume $|s| \leq 8$ by Theorem 2.10, since 1/0 is an exceptional filling. By Proposition 2.6, we must show that each of these links is not a Montesinos link or a Seifert link.

Method 1 (see Section 2.7) can be used to show that none of the $L_{p, q, r}$ are Montesinos knots or links, though it should be noted that the Kauffman polynomial must be employed in a handful of cases, including distinguishing $L_{1,4,-1}$ from $K[1 / 3,2 / 5,-2 / 5]$ and some nonintegral cases, and that the HOMFLYPT polynomial must be employed to distinguish $L_{1,6,-2}$ from $K[-1 / 4,1 / 6,2 / 7]$. In other words, these pairs are not distinguished by their Alexander polynomial and Khovanov homology alone.

Now, consider when $L_{p, q, r}$ is a link. Then it is the union of the unknot and the 2-bridge knot $K[n / m]$, where $n=2 p q-p-2$ and $m=4 p q-2 p-2 q-3 . K[n / m]$ is a torus knot only if $p=1$ and $q=3$ or 4 . In the latter case, $L_{1,4, r}$ is the union of a trefoil and an unknot. If this link is to be a Seifert link, the unknotted component must lie as the core of the torus upon which the trefoil sits. However, the link just described can be distinguished from $L_{1,4, r}$ for all values of $r$ using the Jones polynomial. Concerning $L_{1,3, r}$, exceptional surgeries on the knot $P(-2,3,7)$ are previously well-understood [15].

It only remains to show that $L_{p, q, r}$ is never a torus knot. If $p \neq 1$, this is accomplished by applying Criterion 2.17. For $p=1$, Criterion 2.18 suffices. 


\section{The case of $\left(3,3,\left|q_{3}\right|\right)$}

We now turn our attention to pretzel knots $P\left(q_{1}, q_{2}, q_{3}\right)$ such that $\left|q_{1}\right|=\left|q_{2}\right|=3$. The case of $P\left(3,3, q_{3}\right)$, where $q_{3}>0$ was handled by Ichihara and Jong in [22]. We break up the remaining cases as follows:

(1) $P(3, \pm 3,-2 m)$ with $m \geq 1$

(2) $P(3,3,2 m+1)$ with $m \leq-2$

(3) $P(3,-3,2 m+1)$ with $m \leq-3$ or $2 \leq m$

(4) $P(3,3,2 m,-1)$ with $2 \leq m$

In Cases (2) and (3), it is only necessary to consider integral surgery slopes by Theorem 1.5. Our main result is the following.

Theorem 1.2 Hyperbolic pretzel knots of the form $P(3,3, m)$ or $P(3,3,2 m,-1)$ admit no small Seifert fibered surgeries. Pretzel knots of the form $P(3,-3, m)$, with $m>1$, admit small Seifert fibered surgeries precisely in the following cases:

- $P(3,-3,2)(1)=S^{2}(1 / 3,1 / 4,-3 / 5)$

- $P(3,-3,3)(1)=S^{2}(1 / 2,-1 / 5,-2 / 7)$

- $P(3,-3,4)(1)=S^{2}(-1 / 2,1 / 5,2 / 7)$

- $P(3,-3,5)(1)=S^{2}(2 / 3,-1 / 4,-2 / 5)$

- $P(3,-3,6)(1)=S^{2}(1 / 2,-2 / 3,2 / 13)$

\subsection{Case (1)}

Let $K_{m}^{ \pm}=P(3, \pm 3,-2 m)$. To avoid the redundancy of mirrors, we can restrict to $m>0$. Recall that these knots can only admit nonintegral small Seifert fibered surgeries in the case of $K_{m}^{+}$. Our first result is half of Theorem 1.2 (up to mirroring).

Proposition 4.1 Let $K_{m}^{ \pm}=P(3, \pm 3,-2 m)$ with $m>0$ be hyperbolic. Then, $K_{m}^{ \pm}$ admits no small Seifert fibered surgeries, except in the following three instances:

- $\quad P(3,-3,-2)(-1)=S^{2}(2 / 3,-1 / 4,-2 / 5)$

- $P(3,-3,-4)(-1)=S^{2}(1 / 2,-1 / 5,-2 / 7)$

- $P(3,-3,-6)(-1)=S^{2}(1 / 2,-1 / 3,-2 / 13)$ 


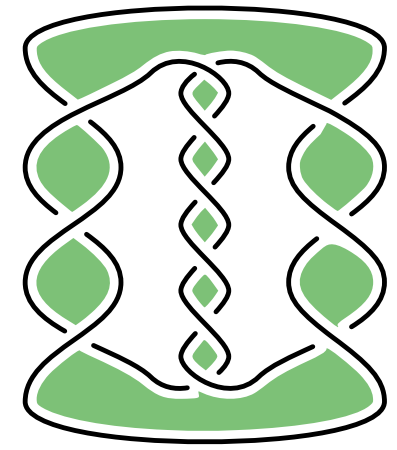

(a)

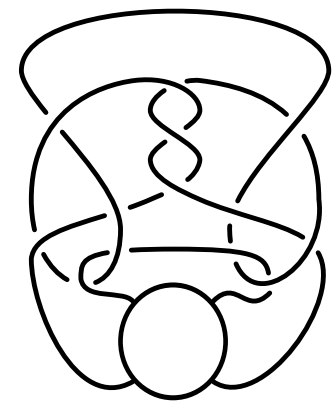

(b)

Figure 12: (a) The knot $K_{m}^{-}=P(3,-3,-2 m)$ (shown here with $m=3$ ) bound punctured Klein bottles; (b) The tangle $\mathcal{T}_{3}^{+}$

As in Section 3, we will proceed in this case by first limiting the possible surgery slopes, then limiting the size of $m$, then using techniques from Section 2.7 to check that small values of $m$ and slopes satisfying the relevant bound do not produce small Seifert fibered spaces (except for the three noted cases). Let $K_{m}^{ \pm}=P(3, \pm 3,-2 m)$ with $m>0$. We begin by observing that $K_{m}^{ \pm}$bounds a punctured Klein bottle. Let $\alpha_{r}^{+}=12-r$ and $\alpha_{r}^{-}=-r$ (again, these are chosen so that $K_{m}^{ \pm}\left(\alpha_{r}\right)$ corresponds to $r$-filling on the corresponding tangle). Then this Klein bottle has boundary slope $\alpha_{0}^{ \pm}$ (see Figure 12(a)). Since surgery along this slope produces a toroidal manifold (as in the previous section), any exceptional surgery slope for $K_{m}^{ \pm}$must be close to $\alpha_{0}^{ \pm}$. In particular, $\Delta\left(\alpha_{r}^{ \pm}, \alpha_{0}^{ \pm}\right) \leq 8$.

Next, we remark that $K_{m}^{ \pm}$is strongly invertible. Let $\mathcal{T}_{m}^{ \pm}$be the resulting quotient tangle, and let $L_{m, r}^{ \pm}=\mathcal{T}_{m}^{ \pm}(r)$.

Lemma 4.2 Suppose $K_{m}^{ \pm}\left(\alpha_{r}\right)$ is a small Seifert fibered space. Then, $m \leq 8$.

Proof Let $L_{m, r}^{ \pm}=\mathcal{T}_{m}^{ \pm}(r)$ and form the tangle $\mathcal{S}_{r}^{ \pm}$by removing a 3-ball containing the $m$-twist box of $L_{m, r}^{ \pm}$(see Figure 13). Let $M_{r}^{ \pm}=\widetilde{S}_{r}^{ \pm}$. Of course, $\mathcal{S}_{r}^{ \pm}(-1 / m)=L_{m, r}^{ \pm}$, and $K_{m}^{ \pm}\left(\alpha_{r}\right)=M_{r}^{ \pm}(1 / m)$. Assume, for a contradiction, that $M_{r}^{ \pm}(1 / m)$ is a small Seifert fibered space for some $m \geq 9$.

Consider the following fillings of $M_{r}^{ \pm}$, which we can easily visualize and verify by looking at the corresponding rational tangle fillings of $\mathcal{S}_{r}^{ \pm}$(see Figure 14):

$$
\begin{aligned}
M_{r}^{ \pm}(1 / m) & =\text { small Seifert fibered space (by assumption) } \\
M_{r}^{-}(0) & =\left(S^{1} \times S^{2}\right) \# L(r, 1) \\
M_{r}^{ \pm}(\infty) & =D^{2}(2,3) \cup_{T^{2}} D^{2}(2,3)
\end{aligned}
$$



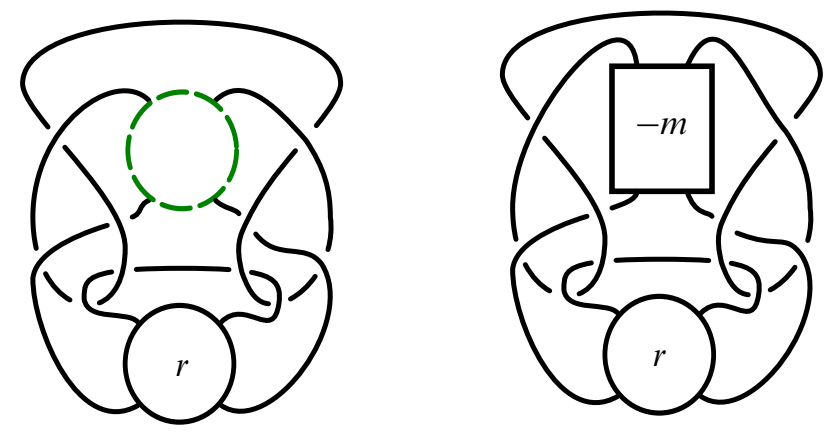

Figure 13: The tangle $\mathcal{S}_{r}^{-}$and the link $L_{m, r}^{-}$

As was argued in Section 3, $M_{r}^{ \pm}$is irreducible (it has distinct irreducible fillings), non-Seifert fibered (it has a non-Seifert fibered, nonreducible filling), and $\partial$-irreducible (it is irreducible and not $S^{1} \times D^{2}$ ). Assume that $M_{r}^{-}$is toroidal, so $M_{r}^{-}=A \cup_{F} B$ with $A$ atoroidal and $\partial M_{r}^{-} \subset B$.

Suppose that $F$ compresses in $M_{r}^{-}(\infty)$. Then, since $\Delta(1 / m, \infty)=|m| \geq 2, B$ is a cable space with cabling slope $\gamma$ satisfying $\Delta(\gamma, \infty)=\Delta(\gamma, 1 / m)=1$. It follows that $\gamma=a \in \mathbb{Z}$, and $|m a-1|=1$. Since $|m| \geq 9, a$ must be zero, so $\gamma=0$. It follows that $B(0)=\left(S^{1} \times D^{2}\right) \# L$, where $L$ is a lens space, and $B(\infty)=B(1 / m)=S^{1} \times D^{2}$. Let $\eta_{0}, \eta_{\infty}$ and $\eta_{1 / m}$, be the slopes of the induced slopes of the meridian of $B$ after the above fillings are performed, so $M_{r}^{-}(\alpha)=A\left(\eta_{\alpha}\right)$ for $\alpha=\infty$ or $1 / m$, and $M_{r}^{-}(0)=A\left(\eta_{0}\right) \# L$. Since $L$ has finite fundamental group, $L=L(r, 1)$ and $A\left(\eta_{0}\right)=S^{1} \times S^{2}$.

Now, since we have $\Delta\left(\eta_{\infty}, \eta_{0}\right) \geq 4$ (by Lemma 2.1), $A$ cannot be hyperbolic, because $\Delta\left(S^{2}, T^{2}\right)=3$. Since $A$ was assumed to be atoroidal, it follows that $A$ must be Seifert fibered. But $A\left(\eta_{\infty}\right)$ is irreducible and not Seifert fibered, so this cannot be. This contradiction means that $F$ cannot compress in $M_{r}^{-}(\infty)$.

So, assume $F$ remains incompressible in $M_{r}^{-}(\infty)$. If $|r|=1$, then $M_{1}^{-}(0)=S^{1} \times S^{2}$. But since $F$ is the unique incompressible torus in a non-Seifert fibered graph manifold, $A=D^{2}(2,3)$. In particular, $M_{1}^{-}(0)=A\left(\eta_{0}\right)=S^{1} \times S^{2}$ is not a possible filling of a trefoil complement.

If $|r|>1$, then since $F$ compresses in fillings at slopes $1 / m$ and 0 , which are at distance one, by Lemma $2.1, B$ is either a cable space or the exterior of a braid in a solid torus. Since $M_{r}^{-}(0)=\left(S^{1} \times S^{2}\right) \# L(r, 1)$, either $B(0)$ or $A\left(\eta_{0}\right)$ is $S^{1} \times S^{2}$. However, this is not possible for such spaces $B$, nor is it possible for $A=D^{2}(2,3)$.

Thus, $M_{r}^{-}$is not toroidal, and so it must be hyperbolic. So, by Theorem 2.11, $\Delta(1 / m, \infty)=|m| \leq 8$, a contradiction that yields the desired result. The reasoning 
is very similar to show that $M_{r}^{+}$must be hyperbolic as well, noting that $M_{r}^{+}(0)=$ $D^{2}(2,2) \cup_{T^{2}} D^{2}(2, r)$ is toroidal for all $r$, and $M_{r}^{+}(1)=S^{2}(1 / 3,-1 / 4,-1 / r)$.
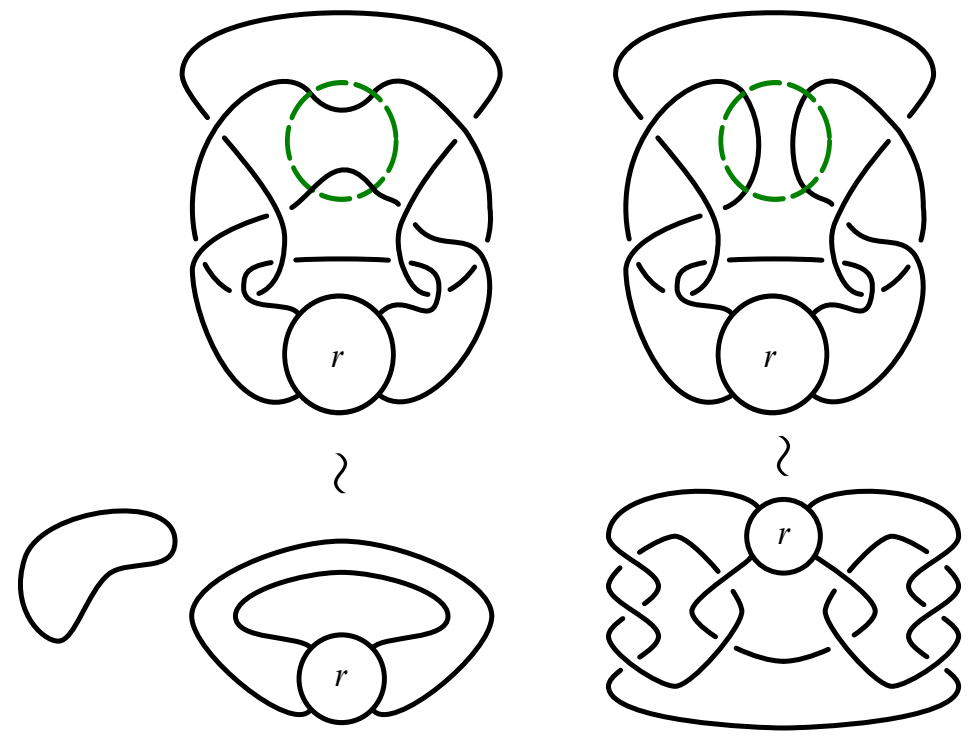

Figure 14: Two interesting fillings of $\mathcal{S}_{r}^{-}$

\subsection{Completing the proof of Proposition 4.1}

By our work above, we can conclude that if $P(3, \pm 3,-2 m)\left(\alpha_{r}^{ \pm}\right)$with $m>0$ admits a small Seifert fibered surgery, then $|r|,|m| \leq 8$. Let $L_{m, r}^{ \pm}=\mathcal{T}_{m}^{ \pm}(r)$. We assume that $r$ is integral for $L_{m, r}^{-}$.

First, consider the links $L_{m, r}^{ \pm}$, which are the union of an unknotted component with a component $J_{m}^{ \pm}=K[2 / 3, \pm 2 / 3,-1 / 2 m]$ (to see this, consider $L_{m, 0}^{ \pm}$, or compare with Figure 26). Because $J_{m}^{ \pm}$is not a torus knot or a 2-bridge knot for any $m$, by Criteria 2.15 and 2.19, we can conclude that $L_{m, r}^{ \pm}$is never a 2-component Seifert link or Montesinos link.

When $L_{m, r}^{ \pm}$is a knot, we see that $2 g\left(L_{m, r}^{ \pm}\right) \neq s\left(L_{m, r}^{ \pm}\right)$, so, by Criterion $2.17, L_{m, r}^{ \pm}$ is never a torus knot. To see that $L_{m, r}^{ \pm}$is never a Montesinos knot, we implement Method 1 (see Section 2.7), accounting for $r$ nonintegral when necessary.

\subsection{Case (2)}

Next, we will consider hyperbolic pretzel knots of the form $K_{m}=P(3,3,2 m+1)$. Here, $K_{m}$ is hyperbolic if $m \neq-1$ or 0 , and if $m$ is positive, then Ichihara and Jong 
have shown that $K_{m}$ admits no Seifert fibered surgeries [22]. The case when $m=-2$ will be covered in Section 4.5, so assume $m \leq-3$. In this section, we prove the following.

Proposition 4.3 A pretzel knot of the form $P(3,3,2 m+1)$ with $m \leq-3$ admits no small Seifert fibered surgeries.

As before, we will first restrict the possible values of $m$ for which $K_{m}$ might admit a Seifert fibered surgery, then rule the remaining cases out by computer. First we note that $K_{m}$ has genus one, so by Theorem 2.12, $|r| \leq 3$ (see Figure 15). Since the three pretzel parameters are all odd, $K_{m}$ cannot admit nonintegral Seifert fibered surgeries by Theorem 1.5. Let $\alpha_{r}=-r$, so that $K_{m}\left(\alpha_{r}\right)=K_{m}(-r)$ will correspond with $\mathcal{T}_{m}(r)$ (the rationally filled quotient tangle), as before.
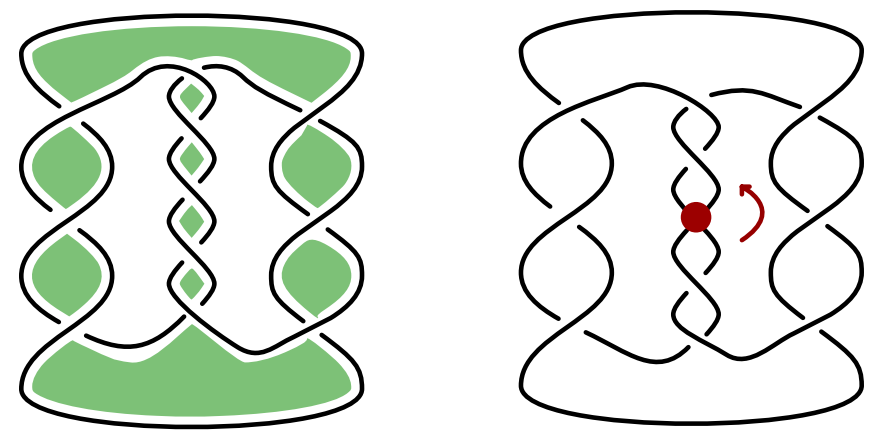

Figure 15: The left figure above shows the pretzel knot $P(3,3,2 m+1)$ as the boundary of a punctured torus, while the right one exhibits the strong inversion of $P(3,3,2 m+1)$ given by rotation. Here, $m=-3$.

Lemma 4.4 If $m \leq-10$, then $K_{m}(r)$ is not a small Seifert fibered space.

Proof In a slight variation of the preceding cases, these knots possess a strong inversion that is a half rotation of the plane. Let $\mathcal{T}_{m}(r)$ be the resulting quotient link, as before (see Figure 16). Again, we form the tangle $\mathcal{S}_{r}$ by removing a ball containing the $(m+1)$-twist region (see Figure 17). If we denote by $N_{r}$ the branched double cover of $S^{3}$ along $\mathcal{S}_{r}$, and assume for a contradiction that $K_{m}(r)$ is a small Seifert fibered space for some $m \leq-10$ and some $r$, then we have the following fillings of $N_{r}$ (see Figure 17). Note that $N_{r}(\infty)=P(3,3,-1)(r)$, so it is simply $r$-surgery on the left 
handed trefoil:

$$
\begin{aligned}
N_{r}(-1 /(m+1)) & =\text { small Seifert fibered space }(\text { by assumption) } \\
N_{r}(0) & =\text { non-Seifert fibered toroidal space } \\
N_{r}(\infty) & =S^{2}(-1 / 2,1 / 3,-1 /(r+6))
\end{aligned}
$$
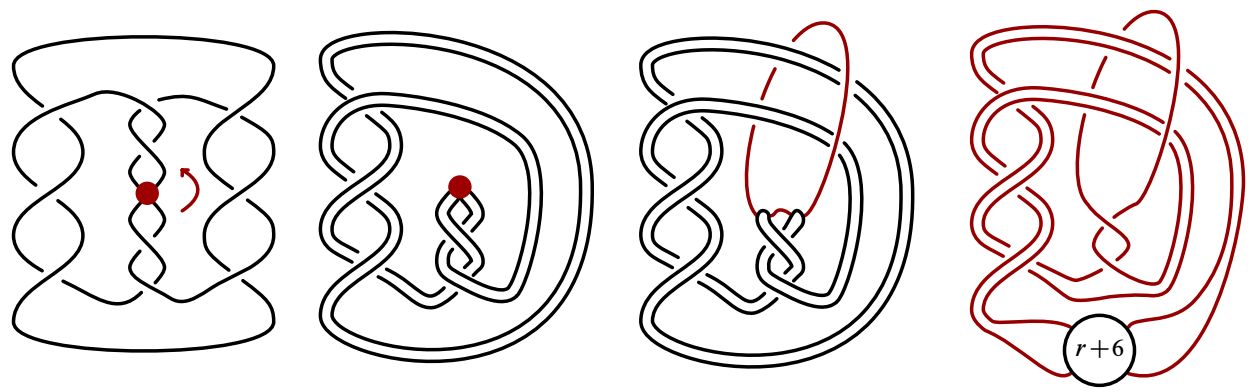

Figure 16: The figures above illustrate how to obtain the quotient tangle $\mathcal{T}_{m}$ by applying the Montesinos trick to the strong inversion of $P(3,3,2 m+1)$ given by rotating the knot $\pi$ radians through its center. Here, $m=-3$.

Since $N_{r}$ has distinct irreducible fillings and a non-Seifert fibered irreducible filling, $N_{r}$ is irreducible, non-Seifert fibered, and $\partial$-irreducible. If $N_{r}$ is toroidal, then since it has atoroidal fillings at distance $\Delta(-1 /(m+1), \infty)=|m+1|>2$, it has as a subspace a cable space with cabling slope $\gamma=0$. This means that $N_{r}(0)$ is either reducible or a lens space, which is a contradiction. It follows that $N_{r}$ is hyperbolic and that $\Delta(-1 /(m+1), \infty)=|m+1| \leq 8$, a contradiction that completes the proof.

\subsection{Completing the proof of Proposition 4.3}

Let $L_{m, r}=\mathcal{T}_{m}(r)$ denote the quotient link described above, and note that it is only necessary to consider $r \in \mathbb{Z}$ here. If $L_{m, r}$ is a link, then we see that it is the union of a trefoil with the knot $J_{m}=K[1 / 2,1 / 3,(m-1) /(2 m-3)]$. Since $m \leq-3$, by assumption, $J_{m}$ is never a torus knot or a 2-bridge knot. It follows that $L_{m, r}$ is never a Seifert link or a Montesinos link, by Criteria 2.19 and 2.15, respectively.

When $L_{m, r}$ is a knot, we see that $\left|\operatorname{Kh}\left(L_{m, r}\right)\right|=6$ and $s\left(L_{m, r}\right)<\operatorname{br}\left(\Delta_{K_{m, r}}(t)\right)$, so $L_{m, r}$ cannot be a Montesinos knot or a torus knot by Criteria 2.14 and 2.17, respectively.

\subsection{Case (3)}

We now consider hyperbolic pretzel knots $K_{m}=P(3,-3,2 m+1)$. We will allow $m$ to be positive or negative, which will allow us to restrict our analysis to positive surgery 


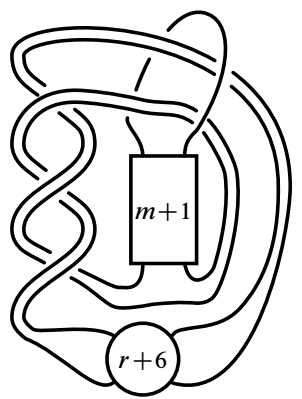

(a)

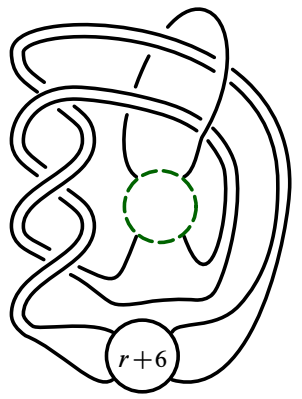

(b)

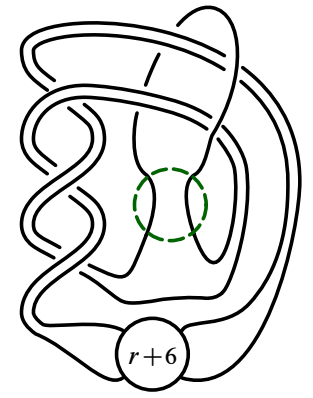

?

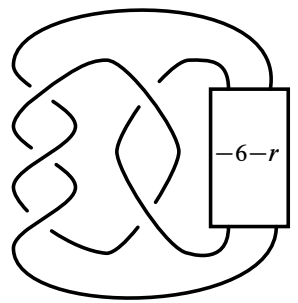

(c)

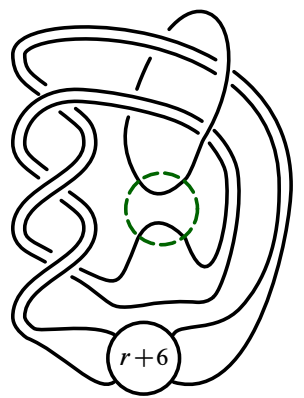

(d)

Figure 17: The first figures above, from left to right, are: (a) the link $\mathcal{T}_{m}\left(-\alpha_{r}\right)$, (b) the tangle $\mathcal{S}_{r}$, (c) the filling $\mathcal{S}_{r}(\infty)$, which is isotopic to $K[-1 / 2,1 / 3,-1 /(6+r)]$, and (d) the fillings $\mathcal{S}_{r}(0)$, whose link complement contains an essential torus.

slopes (which must be integral if they are to be exceptional by Theorem 1.5). These are genus one knots, so $K_{m}(r)$ can only be exceptional if $|r| \leq 3$ by Theorem 2.12 . Thus, we assume $r=1,2$ or 3 .
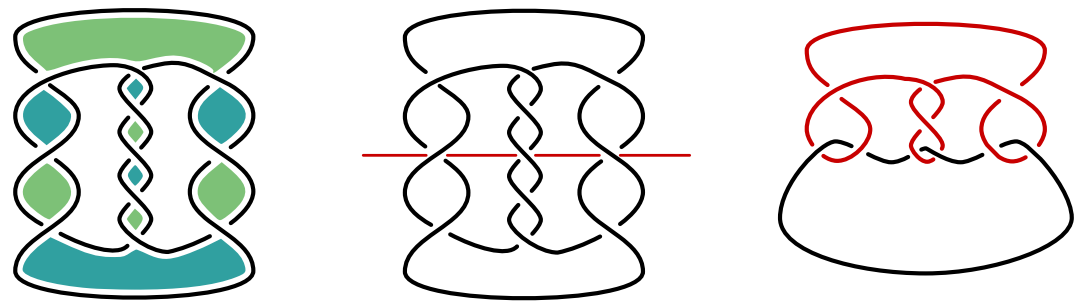

Figure 18: (a) The knot $P(3,-3,2 m+1)$ (here, $m=-3)$, shown as the boundary of a punctured torus, (b) along with the axis of the cycle $f$ of period 2 of the knot and (c) the quotient knot $K_{f}$ (here, the unknot), along with the image of $\operatorname{Fix}(f)$ in the quotient.

These knots are the first that we have encountered with no strong inversion (excepting $P(3,3,-3))$, so we cannot make use of the Montesinos trick. However, $K_{m}$ does have 
cyclic period 2, so we will study the space $K_{m}(r)$ by studying the link $\left(L_{m}\right)_{f}$, which is the image of $\operatorname{Fix}(f) /\langle f\rangle$ in $\left(K_{m}\right)_{f}(r / 2)$ (recall this setup from Section 2.5); see Figure 18.

Proposition 4.5 A hyperbolic pretzel knot of the form $P(3,-3,2 m+1)$ admits a small Seifert fibered surgery precisely in the following instances:

- $P(3,-3,3)(1)=S^{2}(1 / 2,-1 / 5,-2 / 7)$

- $P(3,-3,5)(1)=S^{2}(-1 / 3,-1 / 4,3 / 5)$

Note that the first exceptional surgery was discovered by Song, and the second by Mattman, Miyazaki and Motegi; see [31]. Again, our first task is to restrict the possible values of $m$ for which $K_{m}$ might admit a Seifert fibered surgery, then rule out the remaining cases using knot invariants. We will handle the three cases $r=1,2$ and 3 separately below.

Lemma 4.6 The space $P(3,-3,2 m+1)(1)$ is not a small Seifert fibered space for $|m| \geq 9$.

Proof Assume that $P(3,-3,2 m+1)(1)$ is a small Seifert fibered space with $|m| \geq 9$. As we have seen $K_{m}(1)$ is the branched double cover of $S^{3}$ along the knot $\left(L_{m}\right)_{f}$. We form the tangle $\mathcal{S}$ by removing the $m$-twist box of $\left(L_{m}\right)_{f}$ (see Figure 19). Let $Z=\widetilde{\mathcal{S}}$. Then, we have the following fillings:

$$
\begin{aligned}
Z(-1 / m) & =\text { small Seifert fibered (by assumption) } \\
Z(0) & =S^{2} \times S^{1} \\
Z(-1 / 3) & =D^{2}(2,3) \cup_{T^{2}} D^{2}(2,3) \\
Z(-1 / 2) & =S^{2}(3,5,7) \\
Z(-1) & =S^{2}(2,5,7) \\
Z(\infty) & =S^{2}(2,3,11)
\end{aligned}
$$

It is worth noting that the last four fillings on the list correspond to exceptional fillings of hyperbolic pretzel knots. $P(3,-3,7)(1)=Z(-1 / 3), P(3,-3,5)(1)=Z(-1 / 2)$, $P(3,-3,3)(1)=Z(-1)$ and $P(3,-3,1)(1)=Z(\infty)$. The lattermost is surgery on the rational knot $\mathcal{K}[-2 / 9]$; see Figure 20 .

Because $Z$ has distinct irreducible fillings as well as an irreducible non-Seifert fibered filling $(Z(-1 / 3)$ is a non-Seifert fibered graph manifold), it is impossible for $Z$ to be Seifert fibered, reducible, or $\partial$-reducible. Assume that $Z$ is toroidal, so $Z=A \cup_{F} B$ 


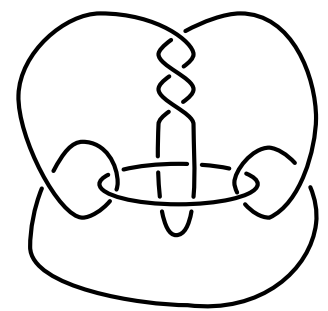

(a)

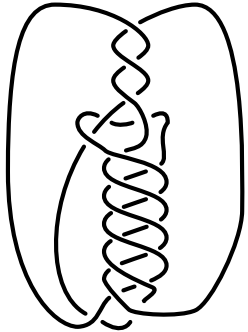

(b)

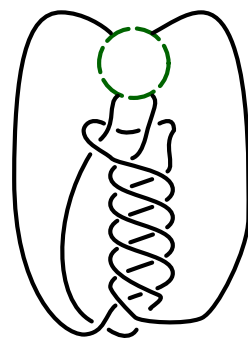

(c)

Figure 19: (a) The link $\left(K_{m}\right)_{f} \cup \operatorname{Fix}(f)$ in the quotient, (b) the knot $\left(L_{m}\right)_{f}$ resulting from $(1 / 2)$-surgery on the unknotted component and (c) the tangle $\mathcal{S}$ formed by removing the $m$-twist area of the knot. Here, $m=-3$.
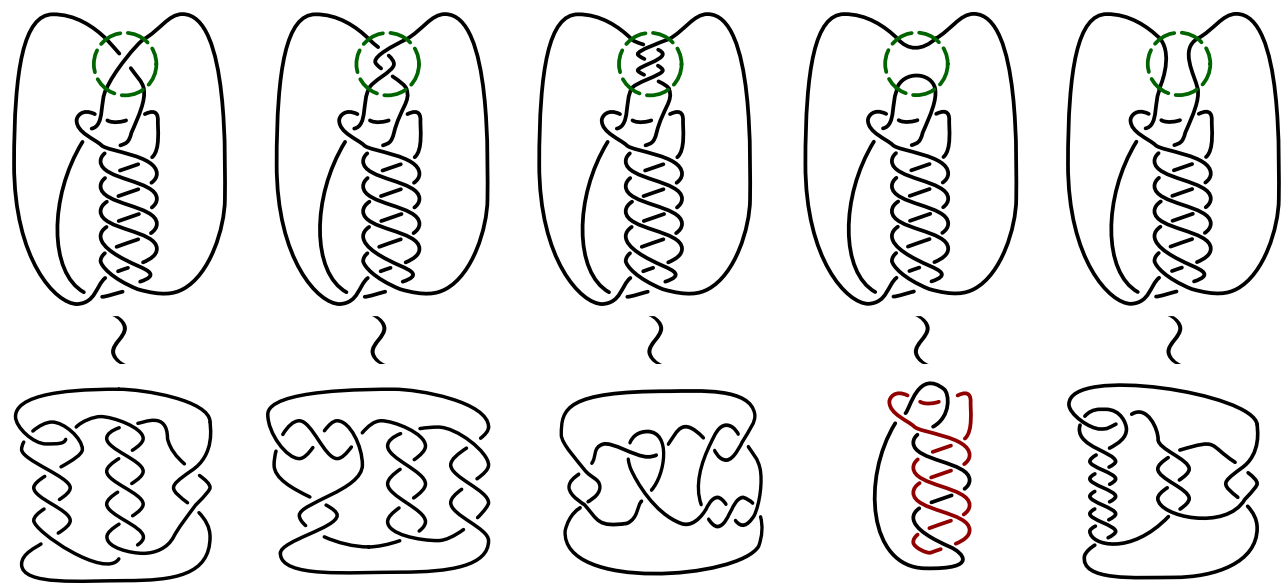

Figure 20: Above, we see five interesting fillings of $\mathcal{S}: \mathcal{S}(1), \mathcal{S}(1 / 2)$, $\mathcal{S}(1 / 3), \mathcal{S}(0)$ and $\mathcal{S}(\infty)$.

with $A$ atoroidal and $\partial Z \subset B$. Then, since $F$ compresses in $Z(-1 / 2), Z(-1)$, and $Z(\infty)$, and $\Delta(1 / 2, \infty) \geq 2, B$ must be a cable space. The cabling slope $\gamma$ is restricted to be distance one from $\infty$ and $-1 / 2$, so $\gamma=0$ or -1 . Since $Z(-1)$ is neither a lens space nor reducible, we cannot have $\gamma=-1$. If $\gamma=0$, then we have $B(0)=\left(S^{1} \times D^{2}\right) \#\left(S^{1} \times S^{2}\right)$, which is not a possible result of filling on a cable space.

It follows that $Z$ is hyperbolic, so $\Delta(-1 / m), \infty) \leq 8$, so $|m| \leq 8$, which gives the desired contradiction.

Proposition 4.7 $P(3,-3,2 m+1)(2)$ is never a small Seifert fibered space for $m \neq 0,-1$. 
Proof We can precede as above, by analyzing the result of 1-surgery on the quotient knot $\left(K_{m}\right)_{f}$. This gives a two-component link in $S^{3},\left(L_{m}\right)_{f}$, such that the double cover of $S^{3}$ branched along $\left(L_{m}\right)_{f}$ is the surgery space $P(3,-3,2 m+1)(2)$. Thus, to show this surgery space is not a small Seifert fibered space, it suffices to show that $\left(L_{m}\right)_{f}$ is neither a Montesinos link of length three with two components nor a Seifert link with two components.

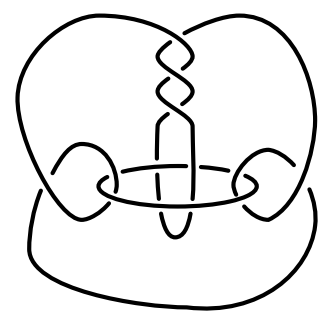

(a)

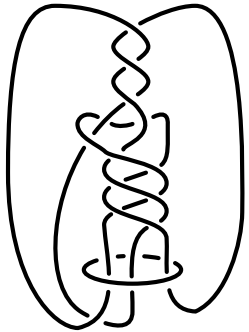

(b)
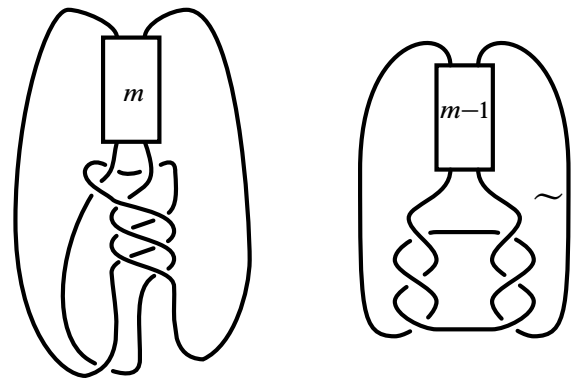

(c)

Figure 21: (a) The link $\left(K_{m}\right)_{f} \cup \operatorname{Fix}(f)$ in the quotient, (b) the link $\left(L_{m}\right)_{f}$ resulting from 1 -surgery on the unknotted component (note that the core of the surgery torus is a component of the resulting link) and (c) the tangle formed by removing the $m$-twist area of the knotted component $J_{m}$ of the resulting link. Here, $m=-3$.

First, we note $\left(L_{m}\right)_{f}$ is the union of the unknot with $J_{m}=K[1 / 3,-1 / 3,1 /(m-1)]$ (see the right half of Figure 21). Since $J_{m}$ is never torus knot, by Criterion 2.19, $\left(L_{m}\right)_{f}$ is never a Seifert link. If $m=0$ or $m=2$, then $J_{0}=K[-2 / 9]$ and $J_{2}=K[2 / 9]$, respectively; otherwise, $J_{m}$ is not a 2-bridge knot, so $\left(L_{m}\right)_{f}$ is not a Montesinos link, by Criterion 2.19 .

If $m=0$, then we are considering 2-surgery on $P(3,-3,1)$, which is $K[2 / 9](2)$. By the classification of Brittenham and $\mathrm{Wu}$ [7], this space is Seifert fibered. If $m=2$, then we are considering the space $P(3,-3,5)(2)$, and if $\left(L_{m}\right)_{f}$ is a Montesinos link, then it has the form $K[x, y, z]$, where $z=2 / 9$ or $4 / 9$ and $x$ and $y$ have even denominator. Now, since $\left(L_{2}\right)_{f}$ has a diagram with 12 crossings, and since the $z$-tangle would contribute 6 crossings, if $\left(L_{2}\right)_{f}$ were to be a two component length three Montesinos link, then the $x$-and $y$-tangles must contribute at most 6 crossings. Without loss of generality, we can assume $x= \pm 1 / 2$ and $y= \pm 1 / 2$ or $\pm 1 / 3$ or $\pm 3 / 4$. However, an easy check shows that the determinant of such Montesinos links cannot be 2 .

Finally, we consider the case of 3-surgery on hyperbolic pretzel knots $K_{m}$ of the type $P(3,-3,2 m+1)$. These knots have period 2 , so we can analyze the surgery 
space $K_{m}(3)$ as the double branched cover of $\left(K_{m}\right)_{f}(3 / 2)$, where $\left(K_{m}\right)_{f}$ is the factor knot $K_{m} / f$ for the self diffeomorphism $f: S^{3} \rightarrow S^{3}$ of order two that preserves $K_{m}$. In this case, $\left(K_{m}\right)_{f}$ is the unknot, so $\left(K_{m}\right)_{f}=-L(3,2)$. Let $L_{m}$ denote the image of Fix $(f) / f$ in the surgery space $\left(K_{m}\right)_{f}(3 / 2)$, ie, $L_{m}$ is the branching set for the double covering. (Note that in our convention $p$-surgery on the unknot is the lens space $-L(p, 1)$.)

Proposition 4.8 $P(3,-3,2 m+1)(3)$ is never a small Seifert fibered space for $m \neq 0,-1$.

Proof By the analysis of [32], the link $L_{m}$ is actually a 2-bridge link, contained in the solid torus that, together with the surgery solid torus, comprises half of the genus- 1 Heegaard splitting of the lens space $-L(3,2)$ (see Figure 22 ). Thus, if we pass to a 3 -fold cover, the lift, $\widetilde{L}_{m}$, of $L_{m}$ will be a length three Montesinos link, contained in one half of the standard genus one Heegaard splitting of $S^{3}$. We now describe how to see this.
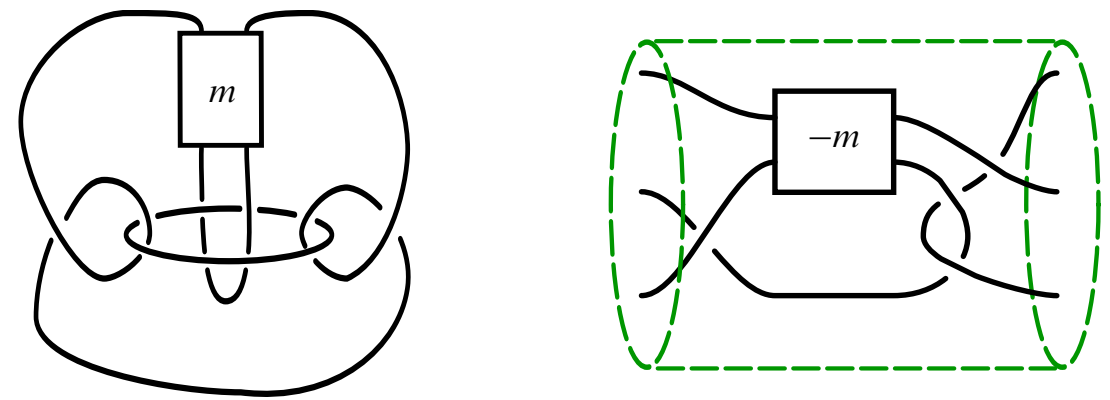

Figure 22: Above, on the left we have the link $\left(K_{m}\right)_{f} \cup \operatorname{Fix}(f) / f$ in the quotient, and, on the right, we have the result of $(3 / 2)$-surgery on the unknotted component: the link $L_{m}$ contained in a solid torus (simply view the knot as lying in the solid torus that comprises the exterior of unknot).

The two solid tori that comprise the splitting of $-L(3,2)$ are attached via a map which sends the meridian of one to a $(-3 / 2)$-curve on the boundary of the other. Passing to the 3 -fold cover changes the image of the attaching map to a $(-1 / 2)$-curve, which gives $S^{3}$. This lift simply triplicates the knotted part of $L_{m}$. However, if we want to think about this lift as a knot in the standard 3-sphere, we must apply a self-diffeomorphism of $S^{3}$ to get the standard Heegaard splitting of $S^{3}$ (ie, where the meridian of one torus is glued along a $(1 / 0)$-curve). This final step introduces two full negative twists of the strands of $L_{m}$. The result is a link $\widetilde{L}_{m}$ in $S^{3}$; see Figure 23. 


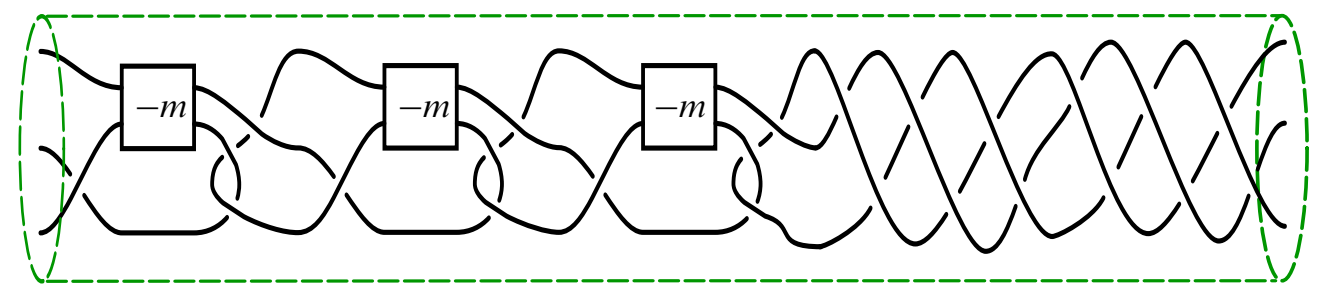

Figure 23: The link $\widetilde{L}_{m}$ in $S^{3}$, which is the triple cover of $L_{m}$ in $-L(3,2)$

Since $P(3,-3,2 m+1)(3)$ has quotient $-L(3,2)$, it has the form $S^{2}(3,3, c)$, where the exceptional fiber of multiplicity $c$ corresponds to the branching locus, $\left(L_{m}\right)_{f}$. It follows that the lift $\tilde{L}_{m}$ should be a Montesinos link of type $K[c, c, c]$. From this, it follows that $c^{2}$ divides the determinant of $\widetilde{L}_{m}$. We can calculate the determinant of this lift to be 49, independent of $m$, so it follows that $c=7$. Thus, $P(3,-3,2 m+1)(3)$ is $S^{2}(3,3,7)$, and $\tilde{L}_{m}=K[a / 7, b / 7, b / 7]$.

By considering the determinant (of the corresponding Montesinos knot), we see that $P(3,-3,2 m+1)(3)$ must have the form $S^{2}(-1 / 3,-1 / 3,5 / 7)$ and we see that $\tilde{L}_{m}=K[-2 ; 5 / 7,5 / 7,5 / 7]$ (being the triple cover of the two bridge knot $K[5 / 7]$ in $S^{1} \times D^{2}$; see [32]). Let $V(q)$ be the Jones polynomial of $K[-2 ; 5 / 7,5 / 7,5 / 7]$. A straightforward calculation gives an expression for the Jones polynomial of $\widetilde{L}_{m}$ :

$$
V_{\widetilde{L}_{m}}(q)= \begin{cases}q^{-3 m-3}(V(q)-1)+1 & \text { if } m \text { is odd, } \\ q^{-3 m}\left(21-V\left(q^{-1}\right)\right)+1 & \text { if } m \text { is even. }\end{cases}
$$

It follows that $\tilde{L}_{m}$ is not $K[-2 ; 5 / 7,5 / 7,5 / 7]$ unless $m=-1$. In this case the knots are the same, which reflects the fact that $P(3,-3,-1)(3)=S^{2}(3,3,7)$; however, this case is not of interest to us. For any other value of $m$, we have shown that $P(3,-3,2 m+1)(3)$ cannot be a small Seifert fibered space.

\subsection{Completing the proof of Proposition 4.5}

It remains to show that the knots $L_{m}=\mathcal{T}_{m}(1)$ are neither Montesinos knots, nor Seifert knots, for $m \in[-8,8] \backslash\{-1,0,1,2\}$ (notice, when $m=1,2$ we $d o$ get small Seifert fibered surgeries, and when $m=-1,0$, we have $P(3,-3, \pm 1)$, which are 2-bridge knots).

In fact, for these knots we have that $s\left(L_{m}\right) \neq 2 g\left(L_{m}\right)$, so they cannot be torus knots by Criterion 2.17. Furthermore, we can apply Method 1 to show that $L_{m}$ is never a Montesinos knot. 


\subsection{Case (4)}

Finally, we consider the case when $K_{m}$ is a hyperbolic pretzel knot of the form $P(3,3,2 m,-1)$ with $m>1$. We note that such knots are often considered to be nonpretzel Montesinos knots. If $m=1$, then $K_{1}=P(-2,3,3)$, and is not hyperbolic. We see that $K_{m}$ has a cyclic of period 2 (see Figure 24) with factor knot $K_{f}=T_{2,3}$ as well as a strong inversion. Since $K_{f}(r / 2)$ must be a lens space surgery on the trefoil, it follows that $\Delta(r / 2,6)=1$. In this case we consider the possibility of nonintegral exceptional surgeries. We note that the link $L_{m, r}=\mathcal{T}_{m}(-r)$ has $4 m+10-r$ half twists; see Figure 26. This can be seen by carefully keeping track of the framing curve throughout the Montesinos trick.

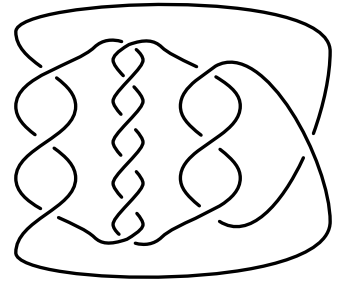

(a)

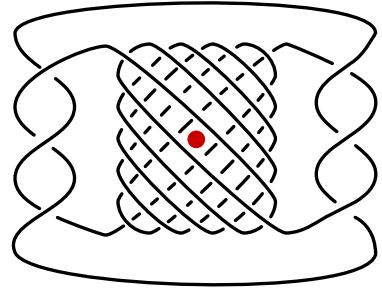

(b)

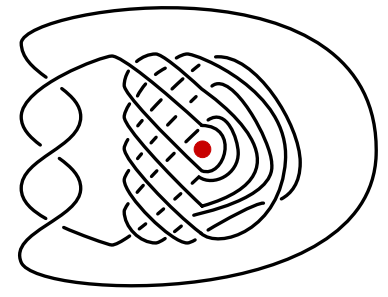

(c)

Figure 24: The Montesinos knot $P(3,3,2 m,-1)$ in (a) standard form, (b) pillowcase form and (c) the factor knot resulting from rotation about the axis perpendicular to the page.
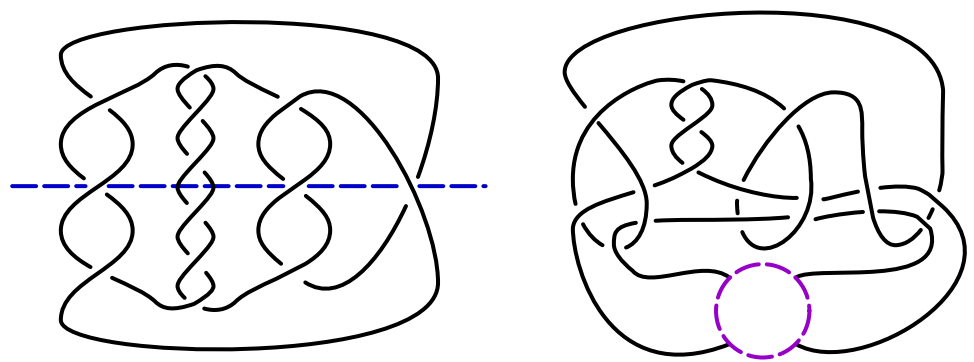

Figure 25: The Montesinos knot $P(3,3,2 m,-1)$, shown with the axis of its strong inversion, and the resulting tangle $\mathcal{T}_{m}$. Here $m=3$.

Proposition 4.9 The Montesinos knots $P(3,3,2 m,-1)$ with $m>1$ admit no small Seifert fibered surgeries.

Proof We begin by noting that we can show that $m \leq 8$ just as we did when dealing with $K_{m}^{+}$, earlier in this section (recall, Figure 14), so we will omit the details. Consider 
the quotient links $L_{m, r}$ obtained via the Montesinos trick. When $L_{m, r}$ is a link, it consists of an unknotted component, together with a component $J_{m}$, which is the knot $K[-1 / 3,-1 / 3,1 / m]$ (see Figure 26).
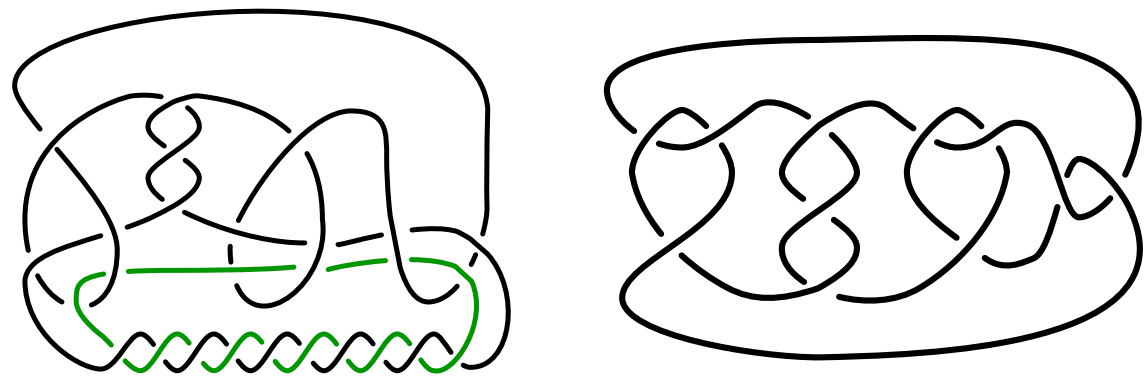

Figure 26: The link $L_{m, r}$ and the component $J_{m}$. Here $m=3$ and $r=12$ (hence, $4 m+10-r=10$ twists).

Since $J_{m}$ is never a torus knot or a 2 -bridge knot (for $m>1$ ), $L_{m, r}$ is never a Seifert link or a Montesinos link, by Criteria 2.19 and 2.15, respectively.

When $L_{m, r}$ is a knot, we see that $\left|\operatorname{Kh}\left(L_{m, r}\right)\right| \geq 4$ and $s\left(L_{m, r}\right)<\operatorname{br}\left(\Delta_{L_{m, r}}(t)\right)$, so, by Criteria 2.14 and 2.17, $L_{m, r}$ is never a Montesinos knot or a torus knot.

\section{The case of $(3,4,5)$}

We next turn our attention to the pretzel knots $P(3, \pm 4, \pm 5)$ and $P(3,4,5,-1)$. We will follow the same program in which we make use of the strong inversion and analyze the quotient link along with its double branched cover. Because these are not infinite families of knots, we do not need to argue to restrict any parameters as we have above. In the case of the length three pretzel knots, since these knots bound punctured Klein bottles at slope $\alpha_{0}$, we only need to consider fillings $\alpha_{r}=\alpha_{0}-r$ at distance at most 8 from $\alpha_{0}$ and from $1 / 0$. Our only task here is to show that the quotient links are not Seifert links nor Montesinos links. In the case of $P(3,-4,5)$, we must consider nonintegral surgeries. The length four pretzel knot $P(3,4,5,-1)$ may also admit nonintegral exceptional surgeries, and, in this case, there is no exceptional surgery by which we can bound the possible surgery slopes. On the other hand, if $P(3,4,5,-1)(r / s)$ is exceptional, then $|s| \leq 4$, since it is known that this pretzel knot is not tunnel number one (see Morimoto, Sakuma and Yokota [35]), and Baker, Gordon and Luecke have recently shown that knots of tunnel number greater than one cannot have nonintegral small Seifert fibered surgery slopes whose denominator is 5 or larger [3]. The pictures corresponding to the analysis of $P(3,4,5,-1)(r / s)$ are nearly 
identical to the diagrams in Figures 25 and 26 (which corresponded to the analysis for $P(3,3,2 m,-1)$ in the previous section; just let $m=2$, and change a $1 / 3$ tangle to a $1 / 5$ tangle) and the reader is encouraged to keep these in mind throughout this section.

Theorem 1.3 The pretzel knots $P(3, \pm 4, \pm 5)$ and $P(3,4,5,-1)$ admit no small Seifert fibered surgeries.

Proof It is shown below, in Lemma 5.2, that the quotient links $L_{r / s}$ corresponding to the surgery spaces $P(3,4,5,-1)(r / s)$ have Khovanov homology of width at least 4 for $|s| \leq 4$ when $L_{r / s}$ is a knot. Thus, Criterion 2.14 suffices to prove that the knots $L_{r / s}$ are not Montesinos knots. Lemma 5.1 shows that $L_{r / s}$ is never a torus knot. If $L_{r / s}$ is a link, then it is the union of the unknot with the Montesinos knot $K[-2 ; 1 / 2,2 / 3,2 / 5]$, which is never a 2-bridge knot or a torus knot, so $L_{r / s}$ is never a Montesinos link or a Seifert link, by Criteria 2.15 and 2.19.

Now, we consider the length three pretzel knots. Let $L_{ \pm, \pm, r}$ be the quotient link resulting from the Montesinos trick, applied to $P(3, \pm 4, \pm 5)$. When $L_{ \pm, \pm, r}$ is a link, $L_{ \pm, \pm, r}=U \cup J$, where $J=K[2 / 3, \pm 1 / 2, \pm 2 / 5]$, which is never the unknot, a two-bridge knot, or a torus knot. Thus, by Criteria 2.15 and $2.19, L_{ \pm, \pm, r}$ is never a Montesinos link with two components or a Seifert link with two components.

When $L_{ \pm, \pm, r}$ is a knot, $2 g\left(L_{ \pm, \pm, r}\right) \neq s\left(L_{ \pm, \pm, r}\right)$, so $L_{ \pm, \pm, r}$ is not a torus knot, by Criterion 2.17, and we can use Method 1 to show that $L_{ \pm, \pm, r}$ is never a Montesinos knot.

Lemma 5.1 $L_{r / s}$ is never a torus knot.

Proof Recall that $|s| \leq 4$, and write $r / s=a / b+n$. A general reference for the facts in this proof is Lickorish [29]. If $|b|=2$ or $|b|=4$, then $L_{r / s}$ has unknotting number one or two. Since the unknotting number of a $(p, q)$-torus knot is $(p-1)(q-1) / 2$, we could only have the trefoil or $T(5,2)$. However, both of these knots are alternating, so they cannot have wide Khovanov homology, as $L_{r / s}$ will be shown to have below. If $a / b=0$ or $b=3$, we can apply the oriented Skein relation to the $n$-twist region of $L_{a / b+n}$ to calculate a recursive formula for the Alexander polynomials $\Delta_{L_{r / s}}(t)$. In general, we write

$$
\Delta_{K}(t)=a_{0}+\sum_{i=1}^{m} a_{i}\left(t^{-i}+t^{i}\right),
$$

and, applying the Skein relation to these knots, we calculate that

$$
\Delta_{L_{a / b+n}}(t)=k_{1}\left(-t^{-1 / 2}\right)^{n}+k_{2}\left(t^{1 / 2}\right)^{n},
$$


where $k_{1}$ and $k_{2}$ are fixed polynomials of small degree, depending on $a / b$ and the sign of $n$. In any event, we see that $a_{m-1}$ for $L_{a / b+n}$ will be constant as $|n|$ increases for a fixed $a / b$. In fact, we can calculate that $\left|a_{m-1}\right|$ takes values $3,2,5,5,7$ and 4 , respectively, for the following cases: $a / b=0$ and $n<0, a / b=0$ and $n>0, a / b=1 / 3$ and $n<0, a / b=1 / 3$ and $n>0, a / b=-1 / 3$ and $n<0$, and $a / b=-1 / 3$ and $n>0$.

If $K$ is a torus knot, then $\left|a_{i}\right| \leq 1$ for all $i$. Thus, $L_{r / s}$ is never a torus knot.

\subsection{A Khovanov homology diversion}

In order to prove that no surgery on $K=P(3,4,5,-1)$ is a small Seifert fibered space, we will argue that the quotient link, $L_{r / s}$ corresponding to $K(r / s)$ has Khovanov cohomology that is too wide when $L_{r / s}$ is a knot and $|s| \leq 4$, ie, $\left|\operatorname{Kh}\left(L_{r / s}\right)\right| \geq 4$.

We will need one important fact about Khovanov cohomology (see, for example, Turner [42], for an overview). Let $D$ be a diagram for a knot, and let $D_{0}$ and $D_{1}$ be the diagrams identical to $D$, except that a single crossing has been resolved as pictured below:

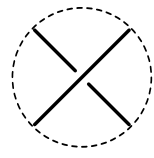

$D$

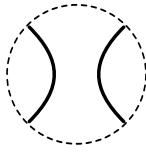

$D_{0}$

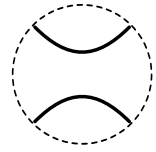

$D_{1}$

Define the value $c$ to be

$c=\left(\right.$ number of negative crossings in $\left.D_{0}\right)-($ number of negative crossings in $D)$.

Then there is a long exact sequence relating the Khovanov cohomology groups:

$$
\longrightarrow \mathrm{Kh}_{j+1}^{i}\left(D_{1}\right) \longrightarrow \mathrm{Kh}_{j}^{i}(D) \longrightarrow \mathrm{Kh}_{j-3 c-1}^{i-c}\left(D_{0}\right) \longrightarrow \mathrm{Kh}_{j+1}^{i+1}\left(D_{1}\right) \longrightarrow
$$

In our examples, one of $D_{0}$ or $D_{1}$ will represent a simple knot type (unknot, Hopf link, trefoil or $(2,4)$-torus link), and so the corresponding Kh will have a small range of support. Outside of this range, there will be isomorphisms between the graded components of $\operatorname{Kh}(D)$ and those of $\operatorname{Kh}\left(D_{1}\right)$ or $\operatorname{Kh}\left(D_{0}\right)$. We will make use of these isomorphisms below.

Lemma 5.2 Let $r / s \in \mathbb{Q}$ with $r / s=a / b+t$ for $a / b \in\{0,1 / 2, \pm 1 / 3, \pm 1 / 4\}$ and $t \in \mathbb{Z}$. Then $\left|\operatorname{Kh}\left(L_{r / s}\right)\right| \geq 4$. 
Proof This proof will be split into cases based on the value of $a / b$. Values of $t$ will be chosen so that $L_{a / b+t}$ is a knot, since the case of a link has already been covered above. Throughout, keep in mind that the diagram $D$ of $L_{r / s}$ is a slight variation on the left side of Figure 26, as mentioned before.

First, assume that $a / b=0$. If $t>0$, then choose one of the negative crossings in the $t$-twist area of a diagram $D$ for $L_{t}$. If $t<0$, form $D$ by creating a pair of opposite crossings next to the $t$-twist area, so that it contains a negative crossing and $t+1$ positive crossings. In either case, the 0 -resolution of the negative crossing, $D_{0}$, is the unknot and the 1-resolution, $D_{1}$, is $L_{t-1}$. In either case, $c=-t-2$. Repeat the process once again, using $D_{1}$ as $D^{\prime}$. Once again, $D_{0}^{\prime}$ is the unknot, but now $D_{1}^{\prime}=L_{t-2}$ and $c^{\prime}=-t-1$. Combining all of this, we have

$$
\mathrm{Kh}_{j}^{i}\left(L_{t}\right) \cong \mathrm{Kh}_{j-2}^{i}\left(L_{t+2}\right) \quad \text { if } i \neq-t-3,-t-2,-t-1
$$

Now, if we refer to Table 1, the second column provides examples of graded components of $\mathrm{Kh}\left(L_{11}\right)$ and $\mathrm{Kh}\left(L_{-9}\right)$ that demonstrate that these knots have wide Khovanov cohomology. But as $|t|$ increases, these graded components are preserved isomorphically (with a grading shift) in $\mathrm{Kh}\left(L_{t}\right)$. It follows that for large values of $|t|, \operatorname{Kh}\left(L_{t}\right)$ is also wide. For small values of $|t|$ (say, $|t| \leq 11$ ), it is easily verified by computer that $\mathrm{Kh}\left(L_{t}\right)$ is wide.

When $a / b=1 / 2$, an identical argument (producing $c$-values of 1 and -3 ) gives us that

$$
\mathrm{Kh}_{j}^{i}\left(L_{1 / 2+t+2}\right) \cong \mathrm{Kh}_{j-4}^{i-2}\left(L_{1 / 2+t}\right) \quad \text { if } i \notin[-3,3] .
$$

One difference here is that $D_{1}$ and $D_{1}^{\prime}$ are the Hopf link, instead of the unknot, so Kh vanishes outside $i \in[-2,2]$. With this in mind, the values in Table 1 give the desired

\begin{tabular}{|c|c|c|c|c|}
\hline Surgery type & $\mathrm{Kh}$ for fixed $r / s$ & General Kh & Diagonals $(j-2 i)$ & Width \\
\hline $\begin{array}{l}\text { Integral } \\
(t<0)\end{array}$ & $\begin{array}{c}\mathrm{Kh}_{1}^{0}\left(L_{-9}\right) \cong \mathbb{Q} \\
\operatorname{Kh}_{21}^{7}\left(L_{-9}\right) \cong \mathbb{Q}\end{array}$ & $\begin{array}{c}\mathrm{Kh}_{-t-8}^{0}\left(L_{t}\right) \cong \mathbb{Q} \\
\mathrm{Kh}_{-t+12}^{7}\left(L_{t}\right) \cong \mathbb{Q}\end{array}$ & $\{-8-t,-2-t\}$ & 4 \\
\hline $\begin{array}{c}\text { Integral } \\
(t>0)\end{array}$ & $\begin{array}{l}\mathrm{Kh}_{-19}^{0}\left(L_{11}\right) \cong \mathbb{Q} \\
\mathrm{Kh}_{-31}^{-10}\left(L_{11}\right) \cong \mathbb{Q}\end{array}$ & $\begin{aligned} \mathrm{Kh}_{-t-8}^{0}\left(L_{t}\right) \cong \mathbb{Q} \\
\mathrm{Kh}_{-t-20}^{-10}\left(L_{t}\right) \cong \mathbb{Q}\end{aligned}$ & $\{-8-t,-t\}$ & 5 \\
\hline $\begin{array}{l}\text { Half integral } \\
(t \text { odd, } t<0)\end{array}$ & $\begin{array}{l}\mathrm{Kh}_{-13}^{-4}\left(L_{1 / 2-5}\right) \cong \mathbb{Q} \\
\mathrm{Kh}_{-21}^{-11}\left(L_{1 / 2-5}\right) \cong \mathbb{Q}\end{array}$ & $\begin{aligned} \mathrm{Kh}_{2 t-3}^{t+1}\left(L_{1 / 2+t}\right) \cong \mathbb{Q} \\
\mathrm{Kh}_{2 t-11}^{t-6}\left(L_{1 / 2+t}\right) \cong \mathbb{Q}\end{aligned}$ & $\{-5,1\}$ & 4 \\
\hline
\end{tabular}
width estimates. 


\begin{tabular}{|c|c|c|c|}
\hline $\begin{array}{l}\text { Half integral } \\
(t \text { even, } t<0)\end{array}$ & $\begin{array}{l}\mathrm{Kh}_{-21}^{-7}\left(L_{1 / 2-6}\right) \cong \mathbb{Q} \\
\mathrm{Kh}_{-29}^{-14}\left(L_{1 / 2-6}\right) \cong \mathbb{Q}\end{array}$ & $\begin{aligned} \mathrm{Kh}_{2 t-9}^{t-1}\left(L_{1 / 2+t}\right) \cong \mathbb{Q} \\
\mathrm{Kh}_{2 t-17}^{t-8}\left(L_{1 / 2+t}\right) \cong \mathbb{Q}\end{aligned}$ & $\{-7,-1\}$ \\
\hline $\begin{array}{l}\text { Half integral } \\
(t \text { odd, } t>0)\end{array}$ & $\begin{array}{c}\mathrm{Kh}_{15}^{7}\left(L_{1 / 2+5}\right) \cong \mathbb{Q}^{2} \\
\operatorname{Kh}_{23}^{14}\left(L_{1 / 2+5}\right) \cong \mathbb{Q}\end{array}$ & $\begin{array}{c}\mathrm{Kh}_{2 t+1}^{t+2}\left(L_{1 / 2+t}\right) \cong \mathbb{Q}^{2} \\
\operatorname{Kh}_{2 t+9}^{t+7}\left(L_{1 / 2+t}\right) \cong \mathbb{Q}\end{array}$ & $\{-5,1\}$ \\
\hline $\begin{array}{c}\text { Half integral } \\
(t \text { even, } t>0)\end{array}$ & $\begin{aligned} \operatorname{Kh}_{7}^{4}\left(L_{1 / 2+6}\right) \cong \mathbb{Q} \\
\operatorname{Kh}_{15}^{11}\left(L_{1 / 2+6}\right) \cong \mathbb{Q}\end{aligned}$ & $\begin{array}{l}\operatorname{Kh}_{2 t-5}^{t-2}\left(L_{1 / 2+t}\right) \cong \mathbb{Q} \\
\operatorname{Kh}_{2 t+3}^{t+5}\left(L_{1 / 2+t}\right) \cong \mathbb{Q}\end{array}$ & $\{-7,-1\}$ \\
\hline $\begin{array}{l}\text { Third integral } \\
\qquad(t<0)\end{array}$ & $\begin{array}{c}\mathrm{Kh}_{-9}^{-4}\left(L_{1 / 3-8}\right) \cong \mathbb{Q} \\
\mathrm{Kh}_{-17}^{-11}\left(L_{1 / 3-8}\right) \cong \mathbb{Q} \\
\operatorname{Kh}_{1}^{1}\left(L_{-1 / 3-6}\right) \cong \mathbb{Q} \\
\mathrm{Kh}_{-13}^{-9}\left(L_{-1 / 3-6}\right) \cong \mathbb{Q}\end{array}$ & $\begin{aligned} \mathrm{Kh}_{-t-17}^{-4}\left(L_{1 / 3+t}\right) \cong \mathbb{Q} \\
\mathrm{Kh}_{-t-25}^{-11}\left(L_{1 / 3+t}\right) \cong \mathbb{Q} \\
\mathrm{Kh}_{-t-5}^{1}\left(L_{-1 / 3+t}\right) \cong \mathbb{Q} \\
\mathrm{Kh}_{-t-19}^{-9}\left(L_{-1 / 3+t}\right) \cong \mathbb{Q}\end{aligned}$ & $\begin{array}{l}\{-t-9,-t-3\} \\
\{-t-7,-t-1\}\end{array}$ \\
\hline $\begin{array}{l}\text { Third integral } \\
\qquad(t>0)\end{array}$ & $\begin{aligned} & \mathrm{Kh}_{-15}^{1}\left(L_{1 / 3+8}\right) \cong \mathbb{Q}^{2} \\
& \mathrm{Kh}_{-25}^{-8}\left(L_{1 / 3+8}\right) \cong \mathbb{Q} \\
& \operatorname{Kh}_{-13}^{1}\left(L_{-1 / 3-8}\right) \cong \mathbb{Q}^{2} \\
& \operatorname{Kh}_{-19}^{-6}\left(L_{-1 / 3-8}\right) \cong \mathbb{Q}\end{aligned}$ & $\begin{array}{l}\mathrm{Kh}_{-t-7}^{1}\left(L_{1 / 3+t}\right) \cong \mathbb{Q}^{2} \\
\mathrm{Kh}_{-t-17}^{-8}\left(L_{1 / 3+t}\right) \cong \mathbb{Q} \\
\mathrm{Kh}_{-t-7}^{1}\left(L_{-1 / 3+t}\right) \cong \mathbb{Q}^{2} \\
\mathrm{Kh}_{-t-11}^{-6}\left(L_{-1 / 3+t}\right) \cong \mathbb{Q}\end{array}$ & $\begin{array}{l}\{-t-9,-t-1\} \\
\{-t-9,-t+1\}\end{array}$ \\
\hline $\begin{array}{l}\text { Fourth integral } \\
(t \text { odd, } t<0)\end{array}$ & $\begin{aligned} \mathrm{Kh}_{-19}^{-8}\left(L_{1 / 4-9}\right) \cong \mathbb{Q} \\
\mathrm{Kh}_{-15}^{-9}\left(L_{1 / 4-9}\right) \cong \mathbb{Q}^{7} \\
\mathrm{Kh}_{-27}^{-9}\left(L_{-1 / 4-9}\right) \cong \mathbb{Q}^{2} \\
\mathrm{Kh}_{-23}^{-10}\left(L_{-1 / 4-9}\right) \cong \mathbb{Q}^{2}\end{aligned}$ & $\begin{aligned} \mathrm{Kh}_{2 t-1}^{t+1}\left(L_{1 / 4+t}\right) \cong \mathbb{Q} \\
\operatorname{Kh}_{2 t+3}^{t}\left(L_{1 / 4+t}\right) \cong \mathbb{Q}^{7} \\
\operatorname{Kh}_{2 t-9}^{t}\left(L_{-1 / 4-t}\right) \cong \mathbb{Q}^{2} \\
\operatorname{Kh}_{2 t-5}^{t-1}\left(L_{-1 / 4-t}\right) \cong \mathbb{Q}^{2}\end{aligned}$ & $\begin{array}{l}\{-3,3\} \\
\{-9,-3\}\end{array}$ \\
\hline $\begin{array}{l}\text { Fourth integral } \\
(t \text { even, } t<0)\end{array}$ & $\begin{array}{c}\mathrm{Kh}_{-29}^{-11}\left(L_{1 / 4-8}\right) \cong \mathbb{Q} \\
\mathrm{Kh}_{-25}^{-12}\left(L_{1 / 4-8}\right) \cong \mathbb{Q}^{7} \\
\mathrm{Kh}_{-19}^{-7}\left(L_{-1 / 4-8}\right) \cong \mathbb{Q} \\
\mathrm{Kh}_{-15}^{-8}\left(L_{-1 / 4-8}\right) \cong \mathbb{Q}^{2}\end{array}$ & $\begin{array}{c}\operatorname{Kh}_{2 t-13}^{t-3}\left(L_{1 / 4+t}\right) \cong \mathbb{Q} \\
\operatorname{Kh}_{2 t-9}^{t-4}\left(L_{1 / 4+t}\right) \cong \mathbb{Q}^{7} \\
\operatorname{Kh}_{2 t-3}^{t+1}\left(L_{-1 / 4-t}\right) \cong \mathbb{Q} \\
\operatorname{Kh}_{2 t+1}^{t}\left(L_{-1 / 4-t}\right) \cong \mathbb{Q}^{2}\end{array}$ & $\begin{array}{l}\{-7,-1\} \\
\{-5,1\}\end{array}$ \\
\hline $\begin{array}{l}\text { Fourth integral } \\
(t \text { odd, } t>0)\end{array}$ & $\begin{aligned} & \operatorname{Kh}_{19}^{8}\left(L_{1 / 4+7}\right) \cong \mathbb{Q}^{2} \\
& \operatorname{Kh}_{15}^{9}\left(L_{1 / 4+7}\right) \cong \mathbb{Q} \\
& \operatorname{Kh}_{9}^{6}\left(L_{-1 / 4+9}\right) \cong \mathbb{Q}^{2} \\
& \operatorname{Kh}_{9}^{9}\left(L_{-1 / 4+9}\right) \cong \mathbb{Q}^{2}\end{aligned}$ & $\begin{aligned} & \mathrm{Kh}_{2 t+5}^{t}\left(L_{1 / 4+t}\right) \cong \mathbb{Q}^{2} \\
& \operatorname{Kh}_{2 t+1}^{t+1}\left(L_{1 / 4+t}\right) \cong \mathbb{Q} \\
& \mathrm{Kh}_{2 t-9}^{t-3}\left(L_{-1 / 4+t}\right) \cong \mathbb{Q}^{2} \\
& \operatorname{Kh}_{2 t-9}^{t}\left(L_{-1 / 4+t}\right) \cong \mathbb{Q}^{2}\end{aligned}$ & $\{-3,-9\}$ \\
\hline $\begin{array}{l}\text { Fourth integral } \\
(t \text { even, } t>0)\end{array}$ & $\begin{aligned} \operatorname{Kh}_{11}^{6}\left(L_{1 / 4+8}\right) \cong \mathbb{Q}^{2} \\
\operatorname{Kh}_{7}^{7}\left(L_{1 / 4+8}\right) \cong \mathbb{Q}^{2} \\
\operatorname{Kh}_{15}^{7}\left(L_{-1 / 4+8}\right) \cong \mathbb{Q} \\
\operatorname{Kh}_{11}^{8}\left(L_{-1 / 4+8}\right) \cong \mathbb{Q}\end{aligned}$ & $\begin{array}{l}\operatorname{Kh}_{2 t-5}^{t-2}\left(L_{1 / 4+t}\right) \cong \mathbb{Q}^{2} \\
\operatorname{Kh}_{2 t-9}^{t-1}\left(L_{1 / 4+t}\right) \cong \mathbb{Q}^{2} \\
\operatorname{Kh}_{2 t-1}^{t-1}\left(L_{-1 / 4+t}\right) \cong \mathbb{Q} \\
\operatorname{Kh}_{2 t-5}^{t}\left(L_{-1 / 4+t}\right) \cong \mathbb{Q}\end{array}$ & $\{-7,-1\}$ \\
\hline
\end{tabular}

Table 1: For each type of $a / b$ (first column), $\operatorname{Kh}\left(L_{a / b+t_{0}}\right)$ can be seen to have width at least 4 (columns 2, 4, and 5). Furthermore, due to the isomorphisms exhibited in Lemma 5.2, these graded components persist (up to consistent grading shifts) for all $|t|>\left|t_{0}\right|$ (column 3), which proves that $\mathrm{Kh}\left(L_{a / b+t}\right)$ always has width at least 4 (columns 3,4, and 5). 
When $a / b= \pm 1 / 3$, the analysis is identical to that of the case when $a / b=0$, except that $D_{0}$ and $D_{0}^{\prime}$ are a trefoil, both of whose $\mathrm{Kh}$ is supported in $i \in[-3,3]$, so we have

$$
\begin{aligned}
\operatorname{Kh}_{j}^{i}\left(L_{1 / 3+t}\right) \cong \mathrm{Kh}_{j-2}^{i}\left(L_{1 / 3+t+2}\right) & & \text { if } i \notin[-t-6,-t+2], \\
\operatorname{Kh}_{j}^{i}\left(L_{-1 / 3+t}\right) \cong \mathrm{Kh}_{j-2}^{i}\left(L_{-1 / 3+t+2}\right) & & \text { if } i \notin[-t-3,-t+5] .
\end{aligned}
$$

When $a / b= \pm 1 / 4$, the analysis is similar to that of the case when $a / b= \pm 1 / 3$, except that $D_{0}$ and $D_{0}^{\prime}$ are both either the $T(2,4)$ or $T(2,-4)$ torus link, both of whose $\mathrm{Kh}$ is supported in $i \in[-4,4]$, so we have

$$
\mathrm{Kh}_{j}^{i}\left(L_{ \pm 1 / 4+t}\right) \cong \mathrm{Kh}_{j+4}^{i+2}\left(L_{ \pm 1 / 4+t+2}\right) \quad \text { if } i \notin[-5,5] .
$$

All these cases are concluded by regarding the information in Table 1, as has been done above.

\section{Nonpretzel Montesinos knots}

In this section, we discuss small Seifert fibered surgery on nonpretzel Montesinos knots. By Wu [46; 45], we need only consider a handful of cases: $K[1 / 3,-2 / 3,2 / 5]$, $K[-1 / 2,1 / 3,2 /(2 a+1)]$ for $a \in\{3,4,5,6$,$\} , and K[-1 / 2,2 / 5,1 /(2 q+1)]$ for $q \geq 1$. We prove the following theorem.

Theorem 1.4 Suppose that $K$ is a nonpretzel Montesinos knot and $K(\alpha)$ is a small Seifert fibered space. Then either $K=K[-1 / 2,2 / 5,1 /(2 q+1)]$ for $q \geq 5$, or $K$ is on the following list and has the described surgeries:

- $K[1 / 3,-2 / 3,2 / 5](-5)=S^{2}(1 / 4,2 / 5,-3 / 5)$

- $K[-1 / 2,1 / 3,2 / 7](-1)=S^{2}(1 / 3,1 / 4,-4 / 7)$

- $K[-1 / 2,1 / 3,2 / 7](0)=S^{2}(1 / 2,3 / 10,-4 / 5)$

- $K[-1 / 2,1 / 3,2 / 7](1)=S^{2}(1 / 2,1 / 3,-16 / 19)$

- $K[-1 / 2,1 / 3,2 / 9](2)=S^{2}(1 / 2,-1 / 3,-3 / 20)$

- $K[-1 / 2,1 / 3,2 / 9](3)=S^{2}(1 / 2,-1 / 5,-3 / 11)$

- $K[-1 / 2,1 / 3,2 / 9](4)=S^{2}(-1 / 4,2 / 3,-3 / 8)$

- $K[-1 / 2,1 / 3,2 / 11](-2)=S^{2}(-2 / 3,2 / 5,2 / 7)$

- $K[-1 / 2,1 / 3,2 / 11](-1)=S^{2}(-1 / 2,-2 / 7,2 / 9)$

- $K[-1 / 2,1 / 3,2 / 5](3)=S^{2}(1 / 2,-1 / 3,-2 / 15)$

- $K[-1 / 2,1 / 3,2 / 5](4)=S^{2}(1 / 2,-1 / 6,-2 / 7)$ 
- $K[-1 / 2,1 / 3,2 / 5](5)=S^{2}(-1 / 3,-1 / 5,3 / 5)$

- $K[-1 / 2,1 / 5,2 / 5](7)=S^{2}(1 / 2,-1 / 5,-2 / 9)$

- $K[-1 / 2,1 / 5,2 / 5](8)=S^{2}(-1 / 4,3 / 4,-2 / 5)$

- $K[-1 / 2,1 / 7,2 / 5](11)=S^{2}(-1 / 3,3 / 4,-2 / 7)$

Question 1.8 Do the Montesinos knots $K[-1 / 2,2 / 5,1 /(2 q+1)]$ with $q \geq 5$ admit small Seifert fibered surgeries?

With the exception of the case noted in the question above, we now prove the list give in Theorem 1.4 is complete. In proving the theorem, we will consider the three types of Montesinos knots involved separately. Note that throughout, we assume $r \in \mathbb{Z}$.

\subsection{The case of $K[1 / 3,-2 / 3,2 / 5]$}

First, consider the case when $K=K[1 / 3,-2 / 3,2 / 5]$. By Wu [51], $K(-4)$ and $K(-6)$ are toroidal, so it suffices to consider $K(r)$ for $-12 \leq r \leq 2$, by Theorem 2.10. Define $L_{r}$, as we have done before (see Figure 27, left side). To show that $L_{r}$ is not a Montesinos knot or link, we apply Method 1. In the case of even $r$, we note that $L_{r}=U \cup T(2,3)$, so if $L_{r}$ is a length three Montesinos link with two components, then one tangle is either a (1/3)-tangle or a (2/3)-tangle. When such a check is performed, precisely one match is found: $K[1 / 3,-2 / 3,2 / 5](-5)$ is a Seifert fibered space, as shown in Theorem 1.4.

To see that $L_{r}$ is never a Seifert link, we simply note that for odd $r, L_{r}$ cannot be a torus knot, by Criterion 2.17, since $\left|s\left(L_{r}\right)\right|<\operatorname{br}\left(\Delta_{L_{r}}(q)\right)$. If $r$ is even, we note that $L_{r}=U \cup T(2,3)$. A priori, $L_{r}$ may be a trefoil union one of its core curves; however, this link has crossing number 7, and the crossing number of $L_{r}$ is at least $\operatorname{br}\left(V_{L_{r}}\right)=10$. Thus, $L_{r}$ is never a two component Seifert link.

\subsection{The case of $K[-1 / 2,1 / 3,2 /(2 a+1)]$}

In the case where $K_{a}=K[-1 / 2,1 / 3,2 /(2 a+1)]$ for $a \in\{3,4,5,6\}$, we note that by $\mathrm{Wu}$ [51] we have the following toroidal fillings: $K_{3}(-2), K_{4}(5), K_{5}(2), K_{5}(-3)$ and $K_{6}(2)$, so we consider surgery slopes $r$ with distance at most 8 from the corresponding toroidal filling.

We proceed as above, considering links and knots $L_{a, r}$ (see Figure 27, right side), to show that $L_{a, r}$ is never a Montesinos knot or link, noting in this case that $L_{a, r}=U \cup J$, where $J$ is $T(2,5)$ if $a=3, T(2,3)$ if $a=4$, and the unknot if $a=5$ or 6 . By 
applying Method 1, we find that the Montesinos links are precisely those stated in Theorem 1.4.

If $r$ is even, and $L_{a, r}$ is a Seifert link, we must have that $L_{3, r}$ is the union of $T(2,5)$, together with a core curve, $L_{4, r}$ is the union of $T(2,3)$, together with a core curve, and $L_{5, r}$ and $L_{6, r}$ have the form $T(2,2 n)$ for some $n$. However, $L_{5, r}$ and $L_{6, r}$ cannot have this form, since they are not alternating, a fact deduced by noticing that $\left|\operatorname{Kh}\left(L_{a, r}\right)\right| \geq 3$. As above, $L_{4, r}$ cannot have the said form because it must have at least 9 crossings. Finally, we see that $L_{3, r}$ has linking number \pm 1 , while $T(2,5)$ has linking number \pm 5 or \pm 2 with its cores. Thus, $L_{a, r}$ cannot be a two component Seifert link.
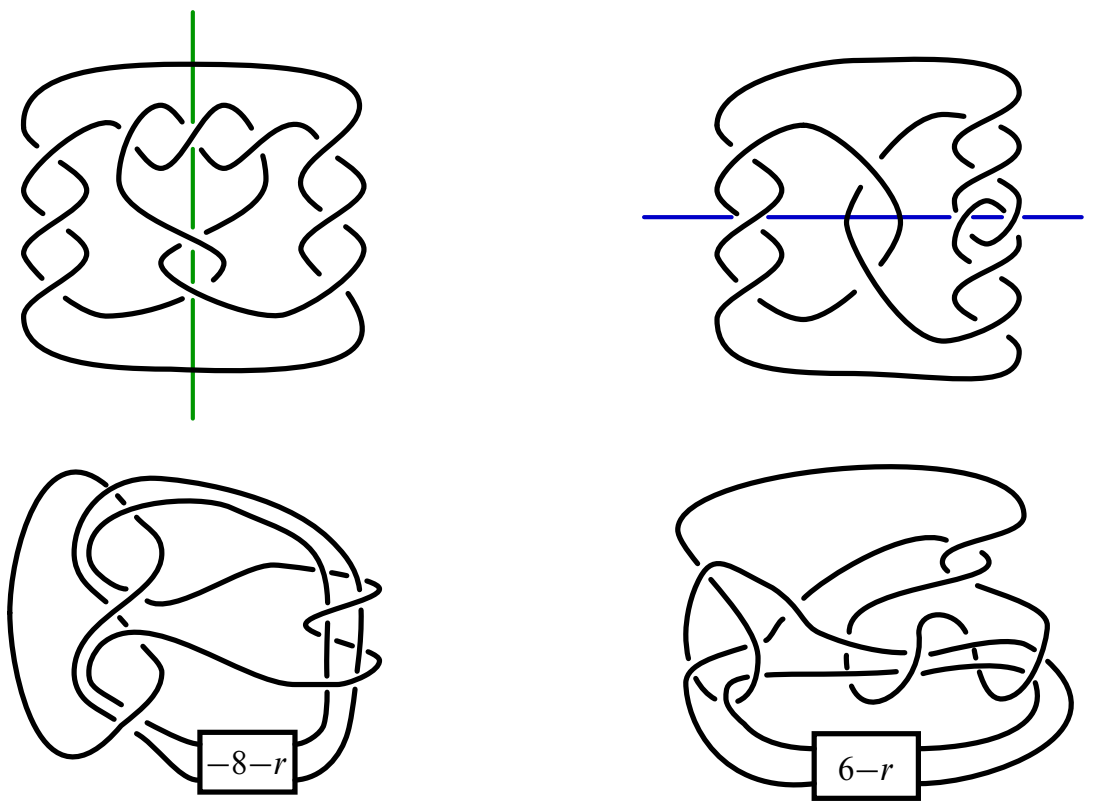

Figure 27: On the left, we have the Montesinos knot $K[1 / 3,1 / 3-3 / 5]$, along with the axis of one of its strong inversions, and the quotient link $L_{r}$. On the right, we have the Montesinos knot $K[-1 / 2,1 / 3,2 / 9]$, along with the axis of its strong inversion, and the quotient link $L_{a, r}$ with $a=4$. In the case of $K[-1 / 2,2 / 5,1 /(2 a+1)]$, we have a picture similar to that on the right.

If $r$ is odd, we can obstruct most of the $L_{a, r}$ from being torus knots by using Criterion 2.17. However, $L_{3,1}$ and $L_{4,1}$ have equal Rasmussen invariant and breadth of Alexander polynomial. The former, in fact, corresponds to a Seifert fibered space, so consider $L_{4,1}$. Since this knot has determinant 1 , if it is to be a torus knot of the 
form $T(a, b)$, both $a$ and $b$ are odd. Furthermore, since it has Rasmussen invariant equal to 10, we have $10=(a-1)(b-1)$, which is impossible if $a$ and $b$ are both odd. Thus, $L_{a, r}$ is never a torus knot.

\subsection{The case of $K[-1 / 2,2 / 5,1 /(2 q+1)]$}

Finally, we are left with the case when $K_{q}=K[-1 / 2,2 / 5,1 /(2 q+1)]$ and $q \geq 1$. By Wu [51], we have the following toroidal fillings: $K_{1}(6), K_{2}(9), K_{3}(12), K_{4}(15)$ so for $q \leq 4$, we can bound the surgery slope $r$, and complete the classification. For larger $q$, we cannot. Furthermore, we obtain no bound on $q$, as we have done above. Below, we argue that when $q \leq 4$, our classification is complete.

If we again consider $L_{q, r}$, we see that for $q \leq 4$ and for any odd $r$, many $L_{q, r}$ have $s\left(L_{q, r}\right)<\operatorname{br}\left(\Delta_{L_{q, r}}(t)\right)$ or $\operatorname{det}\left(L_{q, r}\right)>s\left(L_{q, r}\right)+1$, so they cannot be torus knots, by Criteria 2.17 and 2.18, respectively. However, $\operatorname{det}\left(L_{1, r}\right)=s\left(L_{1, r}\right)+1$ for $r \in\{5,7,9,11,13\}$. So, it is possible that $L_{1, r}=T(2, r)$. However, $\left|\operatorname{Kh}\left(L_{1, r}\right)\right|=3$ for such $r$, so they cannot be alternating knots.

When $r$ is even, we note that $L_{q, r}=U \cup J_{q}$, where $J_{q}$ is the 2-bridge knot $K[2 /(2 q-9)]$. This knot is only a torus knot (or the unknot) if $q=3,4,5$ or 6 , so $L_{q, r}$ can only be a Seifert link for these values, by Criterion 2.19. However, $L_{4, r}$ is never alternating, so it cannot be $T(2,2 n)$, and, $L_{3, r}$ has at least 9 crossings, so it cannot be the union of a trefoil and a core curve.

We show $L_{q, r}$ cannot be a Montesinos knot or link by applying Method 1 . When $r$ is even, we note that one tangle would be a $(2 /(2 q+5))$-tangle or a $((q+3) /(2 q+5))-$ tangle.

\section{References}

[1] T Abe, K Kishimoto, The dealternating number and the alternation number of a closed 3-braid, J. Knot Theory Ramifications 19 (2010) 1157-1181 MR2726563

[2] M M Asaeda, J H Przytycki, Khovanov homology: Torsion and thickness, from: "Advances in topological quantum field theory", NATO Sci. Ser. II Math. Phys. Chem. 179, Kluwer Acad. Publ., Dordrecht (2004) 135-166 MR2147419

[3] K Baker, C M Gordon, J Luecke, Small Seifert fiber spaces from Dehn surgery, in preparation

[4] S Boyer, C M Gordon, X Zhang, Dehn fillings of knot manifolds containing essential once-punctured tori arXiv:1109.5151v3

[5] S Boyer, CM Gordon, $\mathbf{X}$ Zhang, Characteristic submanifold theory and toroidal Dehn filling, Adv. Math. 230 (2012) 1673-1737 MR2927352 
[6] M Brittenham, Exceptional Seifert-fibered spaces and Dehn surgery on 2-bridge knots, Topology 37 (1998) 665-672 MR1604911

[7] M Brittenham, Y-Q Wu, The classification of exceptional Dehn surgeries on 2-bridge knots, Comm. Anal. Geom. 9 (2001) 97-113 MR1807953

[8] G Burde, K Murasugi, Links and Seifert fiber spaces, Duke Math. J. 37 (1970) 89-93 MR0253313

[9] J H Conway, An enumeration of knots and links, and some of their algebraic properties, from: “Computational Problems in Abstract Algebra”, Pergamon, Oxford (1970) 329_ 358 MR0258014

[10] P R Cromwell, Knots and links, Cambridge Univ. Press (2004) MR2107964

[11] M Culler, C M Gordon, J Luecke, P B Shalen, Dehn surgery on knots, Ann. of Math. 125 (1987) 237-300 MR881270

[12] M Dehn, Über die Topologie des dreidimensionalen Raumes, Math. Ann. 69 (1910) 137-168 MR1511580

[13] C Delman, Essential laminations and Dehn surgery on 2-bridge knots, Topology Appl. 63 (1995) 201-221 MR1334307

[14] D Eisenbud, W Neumann, Three-dimensional link theory and invariants of plane curve singularities, Annals Math. Studies 110, Princeton Univ. Press (1985) MR817982

[15] M Eudave-Muñoz, Non-hyperbolic manifolds obtained by Dehn surgery on hyperbolic knots, from: "Geometric topology”, (W H Kazez, editor), AMS/IP Stud. Adv. Math. 2, Amer. Math. Soc. (1997) 35-61 MR1470720

[16] D Futer, M Ishikawa, Y Kabaya, T W Mattman, K Shimokawa, Finite surgeries on three-tangle pretzel knots, Algebr. Geom. Topol. 9 (2009) 743-771 MR2496889

[17] D Gabai, Surgery on knots in solid tori, Topology 28 (1989) 1-6 MR991095

[18] C M Gordon, Small surfaces and Dehn filling, from: "Proceedings of the Kirbyfest", (J Hass, M Scharlemann, editors), Geom. Topol. Monogr. 2 (1999) 177-199 MR1734408

[19] C M Gordon, Dehn surgery and 3-manifolds, from: "Low dimensional topology", (T S Mrowka, P S Ozsváth, editors), IAS/Park City Math. Ser. 15, Amer. Math. Soc. (2009) 21-71 MR2503492

[20] C M Gordon, J Luecke, Knots are determined by their complements, J. Amer. Math. Soc. 2 (1989) 371-415 MR965210

[21] W Heil, Elementary surgery on Seifert fiber spaces, Yokohama Math. J. 22 (1974) 135-139 MR0375320

[22] K Ichihara, ID Jong, Seifert fibered surgery and Rasmussen invariant arXiv: $1102.1117 \mathrm{v} 1$

[23] K Ichihara, I D Jong, Cyclic and finite surgeries on Montesinos knots, Algebr. Geom. Topol. 9 (2009) 731-742 MR2496888 
[24] K Ichihara, I D Jong, Y Kabaya, Exceptional surgeries on (-2, p, p)-pretzel knots, Topology Appl. 159 (2012) 1064-1073 MR2876713

[25] K Ichihara, H Masai, Exceptional surgeries on alternating knots arXiv:1310.3472

[26] S Kang, Examples of reducible and finite Dehn fillings, J. Knot Theory Ramifications 19 (2010) 677-694 MR2646653

[27] M Lackenby, R Meyerhoff, The maximal number of exceptional Dehn surgeries, Invent. Math. 191 (2013) 341-382 MR3010379

[28] S Lee, Lens spaces and toroidal Dehn fillings, Math. Z. 267 (2011) 781-802 MR2776058

[29] W B R Lickorish, An introduction to knot theory, Graduate Texts in Mathematics 175, Springer, New York (1997) MR1472978

[30] W B R Lickorish, M B Thistlethwaite, Some links with nontrivial polynomials and their crossing-numbers, Comment. Math. Helv. 63 (1988) 527-539 MR966948

[31] T Mattman, K Miyazaki, K Motegi, Seifert-fibered surgeries which do not arise from primitive/Seifert-fibered constructions, Trans. Amer. Math. Soc. 358 (2006) 4045-4055 MR2219009

[32] K Miyazaki, K Motegi, Seifert fibering surgery on periodic knots, from: "Proceedings of the First Joint Japan-Mexico Meeting in Topology”, Topology Appl. 121 (2002) 275-285 MR1903696

[33] J M Montesinos, Surgery on links and double branched covers of $S^{3}$, from: "Knots, groups, and 3-manifolds (Papers dedicated to the memory of R. H. Fox)", (LP Neuwirth, editor), Ann. of Math. Studies 84, Princeton Univ. Press (1975) 227-259 MR0380802

[34] J W Morgan, H Bass (editors), The Smith conjecture, Pure and Applied Mathematics 112, Academic Press, Orlando, FL (1984) MR758459

[35] K Morimoto, M Sakuma, Y Yokota, Identifying tunnel number one knots, J. Math. Soc. Japan 48 (1996) 667-688 MR1404816

[36] K Motegi, Dehn surgeries, group actions and Seifert fiber spaces, Comm. Anal. Geom. 11 (2003) 343-389 MR2014880

[37] U Oertel, Closed incompressible surfaces in complements of star links, Pacific J. Math. 111 (1984) 209-230 MR732067

[38] G Perelman, The entropy formula for Ricci flow and its geometric applications arXiv: math.DG/0211159

[39] G Perelman, Finite extinction time for the solutions to the Ricci flow on certain threemanifolds arXiv:math.DG/0307245

[40] G Perelman, Ricci flow with surgery on three-manifolds arXiv:math.DG/0303109 
[41] J Rasmussen, Khovanov homology and the slice genus, Invent. Math. 182 (2010) 419-447 MR2729272

[42] P R Turner, Calculating Bar-Natan's characteristic two Khovanov homology, J. Knot Theory Ramifications 15 (2006) 1335-1356 MR2286127

[43] S Wolfram, The Mathematica ${ }^{\circledR}$ book, 4th edition, Wolfram Media, Champaign, IL (1999) MR1721106

[44] Y-Q Wu, Dehn surgery on knots of wrapping number 2 arXiv:1105.4287

[45] Y-Q Wu, Immersed surfaces and Seifert fibered surgery on Montesinos knots arXiv: $0910.4882 \mathrm{v} 2$

[46] Y-Q Wu, Persistently laminar branched surfaces arXiv:1008.2680v1

[47] Y-Q Wu, Seifert fibered surgery on Montesinos knots arXiv: 12007.0154

[48] Y-Q Wu, Dehn surgery on arborescent knots, J. Differential Geom. 43 (1996) 171-197 MR1424423

[49] Y-Q Wu, Dehn surgery on arborescent knots and links - a survey, Chaos Solitons Fractals 9 (1998) 671-679 MR1628749

[50] Y-Q Wu, Sutured manifold hierarchies, essential laminations, and Dehn surgery, J. Differential Geom. 48 (1998) 407-437 MR1638025

[51] Y-Q Wu, The classification of toroidal Dehn surgeries on Montesinos knots, Comm. Anal. Geom. 19 (2011) 305-345 MR2835882

[52] Y-Q Wu, Exceptional Dehn surgery on large arborescent knots, Pacific J. Math. 252 (2011) 219-243 MR2862149

Department of Mathematics, University of Texas at Austin

1 University Station C1200, Austin, TX 78712, USA

jmeier@math.utexas.edu

http://www .ma.utexas . edu/users/jmeier/

Received: 12 November 2012 Revised: 29 May 2013 